\title{
Amylin Modulates the Mesolimbic Dopamine System to Control Energy Balance
}

\author{
Elizabeth G Mietlicki-Baase*,', David J Reiner', Jackson J Cone², Diana R Olivos', Lauren E McGrath', \\ Derek J Zimmer', Mitchell F Roitman ${ }^{2}$ and Matthew R Hayes*, I \\ 'Translational Neuroscience Program, Department of Psychiatry, Perelman School of Medicine at the University of Pennsylvania, \\ Philadelphia, PA, USA; ${ }^{2}$ Department of Psychology, University of Illinois at Chicago, Chicago, IL, USA
}

\begin{abstract}
Amylin acts in the CNS to reduce feeding and body weight. Recently, the ventral tegmental area (VTA), a mesolimbic nucleus important for food intake and reward, was identified as a site-of-action mediating the anorectic effects of amylin. However, the long-term physiological relevance and mechanisms mediating the intake-suppressive effects of VTA amylin receptor (AmyR) activation are unknown. Data show that the core component of the AmyR, the calcitonin receptor (CTR), is expressed on VTA dopamine (DA) neurons and that activation of VTA AmyRs reduces phasic DA in the nucleus accumbens core (NAcC). Suppression in NAcC DA mediates VTA amylin-induced hypophagia, as combined NACC DI/D2 receptor agonists block the intake-suppressive effects of VTA AmyR activation. Knockdown of VTA CTR via adeno-associated virus short hairpin RNA resulted in hyperphagia and exacerbated body weight gain in rats maintained on high-fat diet. Collectively, these findings show that VTA AmyR signaling controls energy balance by modulating mesolimbic DA signaling.

Neuropsychopharmacology (20I5) 40, 372-385; doi:I0.1038/npp.20I4.I80; published online I3 August 20I4
\end{abstract}

\section{INTRODUCTION}

Amylin is a neuropeptide co-secreted with insulin from pancreatic $\beta$ cells (Johnson et al, 1988; Kahn et al, 1990) that is involved in the regulation of blood glucose (BG; Scherbaum, 1998) and energy balance (for a review, see Lutz, 2005; Mietlicki-Baase and Hayes, 2014). Systemic administration of amylin and amylin agonists such as salmon calcitonin (sCT) and pramlintide reduce food intake and body weight in humans and animal models (Bello et al, 2008; Chapman et al, 2005; Lutz et al, 1994, 2000; Smith et al, 2007), effects mediated by the CNS (Lutz et al, 1995a; Rushing et al, 2000). Previous research has focused heavily on the notion that amylin receptor (AmyR) activation in the area postrema (AP), a hindbrain circumventricular nucleus, is responsible for amylin-induced hypophagia (Lutz et al, 1998; Mollet et al, 2004). Importantly, however, amylin readily crosses the blood-brain barrier (Banks and Kastin, 1998; Banks et al, 1995), and amylin-binding sites are found not only in the AP, but also in other feeding-relevant CNS

\footnotetext{
* Correspondence: Dr EG Mietlicki-Baase, Translational Neuroscience Program, Department of Psychiatry, Perelman School of Medicine at the University of Pennsylvania, 125S. 31 st Street, Philadelphia, PA 19104, USA, Tel: + I 215746 3664, Fax: + I 215573 204I, E-mail: ebaase@mail.med.upenn.edu or Dr MR Hayes, Translational Neuroscience Program, Department of Psychiatry, Perelman School of Medicine at the University of Pennsylvania, 125S. 31st Street, Philadelphia, PA 19104, USA, Tel: + 215573 6070, Fax: + I 215573 2041, E-mail: hayesmr@mail.med.upenn.edu

Received 12 May 20I4; revised 18 June 20I4; accepted 7 July 20।4; accepted article preview online 18 July 2014
}

nuclei (Christopoulos et al, 1995; Paxinos et al, 2004; Sexton et al, 1994). Together with the increasingly recognized distributed nature of energy balance control (Grill, 2006; Grill and Kaplan, 2002), this suggests that amylin may act at multiple sites throughout the brain to regulate feeding and body weight. Recently, the ventral tegmental area (VTA), a mesolimbic nucleus important in the control of food intake and reward (for a review, see Narayanan et al, 2010; Vucetic and Reyes, 2010), was identified as a novel site-of-action for the intake- and body weight-suppressive effects of amylin (Mietlicki-Baase et al, 2013b). However, the long-term physiological relevance and neuronal mechanisms by which VTA AmyR signaling controls energy balance are unknown.

The VTA contains a large population of dopamine (DA) neurons that project to sites throughout the brain that regulate feeding, food reinforcement, and motivated behavior (for a review, see Baik, 2013; Narayanan et al, 2010; Vucetic and Reyes, 2010). In particular, phasic 'spikes' in DA release in the nucleus accumbens (NAc) evoked by aspects of food reward (Brown et al, 2011; Roitman et al, 2004) have been shown to be modulated by feeding-related hormones (Cone et al, 2014) and are sufficient for food reinforcement (Domingos et al, 2011). Given that VTA AmyR activation reduces food intake as well as the motivation to work for a palatable food (Mietlicki-Baase et al, 2013b), these data collectively support the idea that a decrease in NAc DA signaling may be involved in VTA AmyR-mediated hypophagia. However, the ability of VTA AmyR activation to modulate NAc DA signaling to control feeding has not been tested. Furthermore, although previous 
research showed that VTA AmyR signaling acutely reduces food intake, it remains to be determined whether endogenous VTA AmyR signaling is required for the day-to-day control of energy balance. Here, we directly address these questions by examining whether VTA AmyR activation modulates NAc phasic DA neurotransmission, as well as evaluating the requirement of reduced D1/D2 receptor signaling in mediating the intake-suppressive effects of more-palatable and less-palatable food intake following VTA AmyR activation. Using a novel adeno-associated virus short hairpin RNA (AAV-shRNA) construct for the calcitonin receptor (CTR; core component of the AmyR) we also assess the requirement of endogenous VTA AmyR signaling in the long-term control of food intake and body weight in rats maintained on standard chow or high-fat diet (HFD).

\section{MATERIALS AND METHODS}

\section{Subjects}

Adult male Sprague-Dawley rats (Charles River) were housed individually in a temperature- and humiditycontrolled environment on a $12 \mathrm{~h} / 12 \mathrm{~h}$ light-dark cycle. Animals were housed in plastic bins for voltammetric testing and in hanging wire cages for all other studies to allow for accurate measurement of food spillage. Food and water were available ad libitum except where noted. Animal care and use was in accordance with the National Institutes for Health Guide for the Care and Use of Laboratory Animals, and all procedures received approval from the Institutional Animal Care and Use Committee at the University of Pennsylvania or the University of Illinois at Chicago. All behavioral studies utilized a within-subjects, counterbalanced design except where noted.

\section{Drugs}

The AmyR agonist sCT (Bachem) was dissolved in sterile artificial cerebrospinal fluid (aCSF; Harvard Apparatus) for central injections and in sterile $0.9 \% \mathrm{NaCl}$ for peripheral injections. SKF-81297 (Sigma) and quinpirole (Sigma) were dissolved in aCSF. LiCl (Sigma) was dissolved in $0.9 \% \mathrm{NaCl}$. Doses for drugs were selected from the literature (Kanoski et al, 2012; Mietlicki-Baase et al, 2013b; Schmidt and Pierce, 2006; Swanson et al, 1997).

\section{Surgery}

Chronic cannula implantation for feeding studies. Rats were anesthetized by intramuscular (IM) injection of a mixture containing ketamine $(90 \mathrm{mg} / \mathrm{kg})$, xylazine $(2.7 \mathrm{mg} /$ $\mathrm{kg})$, and acepromazine $(0.64 \mathrm{mg} / \mathrm{kg})(\mathrm{KAX})$ and then placed into a stereotaxic apparatus. Analgesia was provided for all surgical procedures $(2 \mathrm{mg} / \mathrm{kg}$ meloxicam). Each rat was stereotaxically implanted with a bilateral guide cannula (26-ga; Plastics One) aimed at the VTA (guide cannula coordinates: $\pm 0.5 \mathrm{~mm}$ lateral to midline, $6.8 \mathrm{~mm}$ posterior to bregma, $6.6 \mathrm{~mm}$ ventral to skull; internal cannula aimed $8.6 \mathrm{~mm}$ ventral to skull). For DA receptor agonist experiments, rats were also stereotaxically implanted with a unilateral guide cannula aimed at the NAc core (NAcC; guide cannula coordinates: $2.5 \mathrm{~mm}$ anterior to bregma, $1.4 \mathrm{~mm}$ left of midline, $4.5 \mathrm{~mm}$ ventral to skull; internal cannula aimed $6.5 \mathrm{~mm}$ ventral to skull). Cannula placements were verified post-mortem by injection of pontamine sky blue (100 nl).

$A A V$ parenchymal delivery. Rats were anesthetized by IM injection of KAX and then placed into a stereotaxic apparatus. Analgesia was provided $(2 \mathrm{mg} / \mathrm{kg}$ meloxicam). Each rat received a bilateral VTA infusion $(250 \mathrm{nl}$ per hemisphere) of an AAV designed to reduce expression of CTR-A/B (CTR-KD), or a control AAV expressing eGFP (CTR-Ctrl); injection coordinates: $\pm 0.5 \mathrm{~mm}$ lateral to midline, $6.8 \mathrm{~mm}$ posterior to bregma, $8.6 \mathrm{~mm}$ ventral to skull.

Surgical preparation for fast-scan cyclic voltammetry (FSCV). Rats were anesthetized by IP injection of ketamine hydrochloride $(100 \mathrm{mg} / \mathrm{kg})$ and xylazine hydrochloride $(10 \mathrm{mg} / \mathrm{kg})$. A guide cannula (Bioanalytical Systems) was implanted dorsal to the right NAcC $(+1.3 \mathrm{~mm}$ anterior and $+1.5 \mathrm{~mm}$ lateral to bregma, $-2.5 \mathrm{~mm}$ ventral to skull). An infusion cannula (Plastics One) was also implanted aimed at the VTA: 26-ga $11 \mathrm{~mm}$ cannula (C315), $-5.8 \mathrm{~mm}$ anterior and $+2.9 \mathrm{~mm}$ lateral to bregma, $-6.5 \mathrm{~mm}$ ventral to skull, angled $15^{\circ}$ away from midline. VTA coordinates were chosen to maximize the likelihood of affecting VTA neurons that project to the NAcC (Ikemoto, 2010). A chlorinated silver reference electrode was placed in left forebrain. Stainless steel skull screws and dental cement secured implants to the skull.

\section{VTA Colocalization of the CTR and Tyrosine Hydroxylase}

Rats $(n=6)$ maintained on HFD were deeply anesthetized and transcardially perfused with $0.1 \mathrm{M}$ phosphate-buffered saline (PBS; $\mathrm{pH}$ 7.4) followed by $4 \%$ paraformaldehyde in $0.1 \mathrm{M}$ PBS. Brains were removed from the crania and postfixed in $4 \%$ paraformaldehyde for $6 \mathrm{~h}$, then stored in $20 \%$ sucrose in $0.1 \mathrm{M} \mathrm{PBS}$ at $4{ }^{\circ} \mathrm{C}$ overnight. Coronal VTAcontaining sections $(30 \mu \mathrm{m})$ were sliced and collected using a cryostat and stored in cryoprotectant at $-20{ }^{\circ} \mathrm{C}$ until the start of immunohistochemistry (IHC). Briefly, tissue was washed with $0.1 \mathrm{M}$ PBS followed by $50 \%$ ethanol to remove the cryoprotectant, followed by washes in $1 \%$ sodium borohydride and $0.3 \% \mathrm{H}_{2} \mathrm{O}_{2}$. Sections were incubated in blocking solution ( $5 \%$ normal donkey serum; Jackson Immunoresearch) followed by $16-\mathrm{h}$ incubation in rabbit anti-CTR primary antibody (1:1000; Abcam) at room temperature. Tissue was then incubated in donkey antirabbit fluorescent secondary antibody (AlexaFluor 488, $1: 222$; Jackson Immunoresearch) for $2 \mathrm{~h}$. Next, sections were then incubated in rabbit anti-tyrosine hydroxylase (TH) primary antibody (1:1000; Cell Signaling) at room temperature for $16 \mathrm{~h}$, followed by 2 -h incubation in donkey anti-rabbit fluorescent secondary antibody (AlexaFluor 594, 1:500; Jackson Immunoresearch) for $2 \mathrm{~h}$. Sections were mounted and visualized using fluorescence microscopy (Nikon 80i; NIS-Elements AR 3.0) at $\times 20$ magnification. CTR and/or TH immunoreactivity were semiquantified from VTA-containing coronal sections between -5.2 and -6.8 from bregma, using a rat brain atlas (Paxinos and Watson, 2006). 
Modulation of NAcC Phasic DA Release by VTA AmyR Activation

Rats $(n=9)$ had ad libitum access to water but were food (chow) restricted to approximately $90 \%$ of their free-feeding body weight during training and testing. All training and experimental sessions took place during the light phase in standard operant chambers (Med Associates) with a food receptacle and magazine for the delivery of single $45 \mathrm{mg}$ sugar pellets $(3.58 \mathrm{kcal} / \mathrm{g}$; BioServ). Rats were trained to retrieve sugar pellets that were delivered with a random inter-trial interval (delivery interval range: $30-90 \mathrm{~s}$; mean: $60 \pm 8.2 \mathrm{~s}$ ). Following 5 days of training, rats were surgically prepared for FSCV. After returning to pre-surgery body weight, rats were retrained for 2 days before the experimental session.

During an experimental session, rats were placed into operant chambers as above. FSCV in awake and behaving rats and analyte identification and quantification have been extensively described (Cone et al, 2013; Phillips et al, 2003). Briefly, a micromanipulator containing a glass-insulated carbon fiber ( $\sim 75 \mu \mathrm{m}$; Goodfellow USA) (recording) electrode was inserted into the NAcC guide cannula. The recording electrode was then lowered into NAcC and locked into place. A FSCV headstage (University of Washington EME Shop) was used to tether the rat, apply voltage changes and measure resultant current changes. The electrode voltage was held at $-0.4 \mathrm{~V}$ and ramped in a triangular fashion $(-0.4$ to +1.3 to $-0.4 \mathrm{~V} ; 400 \mathrm{~V} / \mathrm{s})$ at $10 \mathrm{~Hz}$. In addition, a 33-ga injector connected to a $10 \mu \mathrm{l}$ Hamilton syringe was inserted into the VTA infusion cannula ( $2 \mathrm{~mm}$ projection). To verify that food reward reliably evoked phasic DA release, a single sugar pellet was delivered. If this failed to evoke DA release, the electrode was advanced $0.16 \mathrm{~mm}$ and the process was repeated.

Once a stable release site was confirmed, the experimental session began. Electrochemical data were synced with video and recorded during the entire session. After 10 pellets (mid-session), an infusion pump was activated to deliver intra-VTA sCT $(0.04 \mu \mathrm{g})$ or vehicle $(100 \mathrm{nl} \mathrm{aCSF})$. After the recording session, electrodes were removed; rats were disconnected from the headstage and returned to their home cage. Ad libitum chow intake was monitored for $30 \mathrm{~min}$. Following experiments, all recording electrodes were calibrated in a flow-cell (Sinkala et al, 2012) to convert detected current to concentration. The average calibration factor for all electrodes used in these experiments was $44.58 \pm 4.0 \mathrm{nM} / \mathrm{nA}$.

All rats retrieved and ingested all pellets before and after pharmacological manipulations. Individual trials were background-subtracted and DA concentration during the $10 \mathrm{~s}$ before to $10 \mathrm{~s}$ after pellet retrieval was extracted from voltammetric data using principal component analysis (Heien et al, 2004). We calculated peak DA concentration evoked by pellet retrieval by finding the maximum DA concentration during the $1 \mathrm{~s}$ before to $1 \mathrm{~s}$ after retrieval of each individual pellet. These values were averaged and compared before (baseline) and after VTA infusions to obtain a measure of percent change in peak DA.

Following completion of experiments, rats were deeply anesthetized with sodium pentobarbital $(100 \mathrm{mg} / \mathrm{kg})$ and a small electrolytic lesion was made at the voltammetry recording site using a polyimide-insulated stainless steel electrode (A-M Systems). Rats were then transcardially perfused with cold $0.01 \mathrm{M}$ PBS followed by $10 \%$ buffered formalin solution (Sigma). Brains were removed and stored in formalin for $24 \mathrm{~h}$ and transferred to $30 \%$ sucrose in $0.1 \mathrm{M}$ phosphate buffer (PB). All brains were sectioned at $40 \mu \mathrm{m}$ on a cryostat. NAc sections were mounted and lesion locations were verified using light microscopy (Paxinos and Watson, 2006). All recordings were confirmed to be from the NAcC. In addition, cannula tips from all animals were confirmed to be in the VTA using light microscopy.

\section{Feeding and Body Weight Effects of NAcC DA Receptor Agonists}

Separate groups of chow-maintained rats with NAcC cannulae were given unilateral intra-NAcC injections of either the D1 receptor agonist SKF-81297 (0, 0.1, 0.6, $1.2 \mu \mathrm{g}$; $n=6)$, the D2 receptor agonist quinpirole $(0,1.5,3,6 \mu \mathrm{g}$; $n=6$ ), or a combination of SKF-81297 and quinpirole (1.2 and $6 \mu \mathrm{g}$, respectively; $n=6$ ), just before the onset of the dark phase. For all experiments, vehicle was $200 \mathrm{nl}$ aCSF. Chow intake and body weight change were measured over the 24-h post-injection.

We also tested the effects of intra-NAcC delivery of SKF81297 and quinpirole on HFD intake by conducting similar studies in HFD-maintained rats. Briefly, one group of rats $(n=12)$ received unilateral intra-NAcC injections of SKF$81297(1.2 \mu \mathrm{g})$, quinpirole $(6 \mu \mathrm{g})$, or vehicle ( $200 \mathrm{nl} \mathrm{aCSF})$; a second group of HFD-maintained animals $(n=8)$ received NAcC injections of the combination of SKF-81297 and quinpirole ( 1.2 and $6 \mu \mathrm{g}$, respectively) or vehicle $(200 \mathrm{nl}$ aCSF) just before the onset of the dark phase. HFD intake and body weight were measured for 24 -h post-injection.

\section{Energy Balance Effects of VTA AmyR Activation in Combination with NAcC DA Receptor Agonism}

Just before the onset of the dark phase, chow-maintained rats $(n=10)$ with VTA and NAcC cannulae were given a unilateral intra-NAcC injection containing a mixture of SKF-81297 and quinpirole (1.2 and $6 \mu \mathrm{g}$, respectively) or vehicle ( $200 \mathrm{nl}$ aCSF), followed by a unilateral VTA injection of sCT $(0.04 \mu \mathrm{g})$ or its vehicle $(100 \mathrm{nl} \mathrm{aCSF})$. Subsequent chow intake was measured at $1,3,6$, and $24 \mathrm{~h}$, and 24 -h body weight change was calculated. A separate group of animals $(n=12)$ maintained on HFD ( $60 \% \mathrm{kcal}$ from fat) were given the same NAcC and VTA drug treatments, and $\operatorname{HFD}$ intake $(1,3,6$, and $24 \mathrm{~h})$ and 24 -h body weight change were assessed.

\section{Effect of VTA AmyR Activation on Conditioned Taste Avoidance (CTA)}

A two-bottle CTA test was performed as described previously (Garcia et al, 1955; Kanoski et al, 2012). Briefly, rats were habituated to a water deprivation schedule for 7 days in which water access was given once daily for $90 \mathrm{~min}$, $2 \mathrm{~h}$ after the onset of the light phase. Food was available ad libitum. After habituation, rats were assigned to treatment groups: (1) $0.15 \mathrm{M} \mathrm{LiCl}$, IP $(n=8)$; (2) $0.04 \mu \mathrm{g}$ sCT, intra-VTA $(n=9)$; (3) $0.4 \mu \mathrm{g} \mathrm{sCT}$, intra-VTA $(n=6)$. 
The vehicle for IP injection was $1 \mathrm{ml} / \mathrm{kg} 0.9 \% \mathrm{NaCl}$, while the vehicle for VTA injections was $100 \mathrm{nl}$ aCSF. CTA training consisted of two training days. On each training day during the normal 90-min fluid access period, rats were given access to two burettes containing the same flavor of Kool-Aid (either cherry or grape, flavors equally preferred (Lucas and Sclafani, 1996); $0.14 \mathrm{oz}$ Kool-Aid mix, $10 \mathrm{~g}$ saccharin, 31 water). Immediately after flavor exposure, each rat received IP or intra-VTA injection as described above. Drug treatments were counterbalanced across training days and for paired flavor. Each training day was succeeded by a noninjection day where water was available during the 90-min fluid access period. Two days after the second training day, animals were presented with both flavors (one flavor per burette) during the 90 -min fluid access period. Fluid intake was recorded after $45 \mathrm{~min}$, at which point the side of flavor presentation was switched to avoid side preference. Fluid intake was recorded again at $90 \mathrm{~min}$.

\section{Effect of VTA AmyR Activation on Glycemic Control}

Rats $(n=7)$ were deprived of food overnight $(16 \mathrm{~h})$ to ensure an empty gastrointestinal tract. Oral glucose tolerance testing was conducted mid-light phase; food remained unavailable for the duration of the test. First, a small sample of tail tip blood was obtained from each rat and a standard glucometer (Accucheck, Roche Diagnostics) was used to measure baseline BG values (time $=-20 \mathrm{~min}$ ). Immediately after baseline BG was assessed, each rat received a unilateral intra-VTA injection of sCT $(0,0.004,0.04$, or $0.4 \mu \mathrm{g}$ in $100 \mathrm{nl}$ $\mathrm{aCSF})$. Twenty minutes after VTA injection (time $=0 \mathrm{~min}$ ), BG was measured again, and each rat received a glucose load via oral gavage $(25 \% ; 2 \mathrm{~g} / \mathrm{kg})$. Subsequent $\mathrm{BG}$ readings were taken at 20, 40,60, and 120-min post-glucose load. A minimum of $72 \mathrm{~h}$ separated test sessions.

\section{Meal Pattern Effects of VTA AmyR Activation on HFD Intake}

Rats $(n=10)$ were housed in a custom-built automated feedometer system. Each feedometer consisted of a hanging wire mesh cage with a small access hole leading to a food cup resting on an electronic scale. Food cup weights were recorded by computer software (LabView) every $10 \mathrm{~s}$ for $24 \mathrm{~h}$. Rats were maintained on HFD (60\% kcal from fat; Research Diets) before and throughout testing. Just before dark phase onset, each rat received a unilateral VTA injection of $\operatorname{sCT}(0.01,0.04,0.4 \mu \mathrm{g})$ or vehicle $(100 \mathrm{nl}$ aCSF $)$ before receiving access to preweighed HFD. Food intake and body weight change were monitored at 24 -h post-injection. For meal pattern analyses, a meal was defined as at least $0.25 \mathrm{~g}$ of food intake and a minimum of $10 \mathrm{~min}$ between feeding bouts (Mietlicki-Baase et al, 2013a, b).

\section{Development of AAV for CTR Knockdown}

To create a shRNA-AAV targeting CTR-A/B, we screened commercially available potential sequences for reduced CTR expression (TG712173; Origene) in an in vitro model. Using a commercially available rat immortalized hypothalamic neuronal cell line (R-19; Cedarland Labs), a plasmid designed to overexpress CTR (NM_053816; Origene) was transiently transfected into R-19 cells either alone or in combination with a plasmid sequence to reduce CTR expression. The most robust knockdown of overexpressed CTR was obtained using the following sequence: $\left(5^{\prime}\right.$-GAT CGTCCAGTTCTTCAGGCTCCTACCAATCTCATCAAGAGT GAGATTGGTAGGAGCCTGAAGAACTGGATTTTTT-3' ${ }^{\prime}$ ) targeting CTR-A/B. This sequence was packaged into an AAV vector (serotype I; titer $=5.3 \mathrm{e} 12$ ) designed to express shRNA-CTR-A/B driven by the cytomegalovirus promoter, as well as enhanced green fluorescent protein (eGFP) for visualization. An eGFP-expressing empty vector AAV (titer $=5.0 \mathrm{e} 11)$ was used as a control. To confirm the ability of the AAV to reduce CTR expression in vivo, chowmaintained rats received bilateral, intra-VTA AAV infusion as described above (CTR-KD, $n=5$; CTR-Ctrl, $n=4$ ). Three weeks after viral injection, rats were killed, and brains were rapidly removed from the crania and flash-frozen in $-70{ }^{\circ} \mathrm{C}$ isopentane. To evaluate AAV knockdown efficacy via quantitative real-time PCR (qPCR), $1 \mathrm{~mm}$ micropunches of VTA tissue were collected bilaterally from each brain. qPCR was performed as described previously (Mietlicki-Baase et al, 2013b). CTR-A and -B levels were quantified, with rat GapDH (VIC-MGB) serving as an internal control. PCR reactions for CTR were completed with Taqman gene expression kits (CTR-A: Rn01526770_m1; CTR-B: Rn01526768_m1; GapDH: Rn01775763_g1) and PCR reagents (Applied Biosystems). Analysis of samples was conducted using an Eppendorf Mastercycler ep realplex2. The comparative threshold cycle method (Bence et al, 2006) was used for relative mRNA expression calculations. Correct placement of AAV injections was verified by collecting a VTA-containing coronal section $(30 \mu \mathrm{m})$ from each brain and visualizing eGFP (Figure 6c).

\section{Effect of VTA CTR Knockdown on Acute Response to IP sCT}

Chow-maintained rats were given bilateral intra-VTA AAV infusion (CTR-KD, $n=9$; CTR-Ctrl, $n=7$ ). After surgery, approximately 3 weeks elapsed before initiating behavioral testing to ensure full viral expression. Each rat received an IP injection of sCT $(2 \mu \mathrm{g} / \mathrm{kg})$ or vehicle $(1 \mathrm{ml} / \mathrm{kg}$ sterile $0.9 \%$ $\mathrm{NaCl})$ just before the onset of the dark phase. Chow intake $(1,3,6,24 \mathrm{~h})$ and 24 -h body weight gain were measured.

\section{Long-term Effect of VTA CTR Knockdown on Energy Balance}

Separate rats were maintained on chow or on HFD for 1 week before surgical intra-VTA injection of AAV as described above. Within each dietary condition, half of the rats received VTA injection of AAV to knock down expression of CTR (CTR-KD; chow, $n=7$; HFD, $n=7$ ) and the remaining animals were injected with the AAV control vector (CTR-Ctrl; chow, $n=7$; HFD, $n=7$ ). Food intake (accounting for spillage) and body weight were measured before surgery to make baseline comparisons between treatment groups. Beginning post-surgery day 1, measurements continued every $48 \mathrm{~h}$ for a total of 30 days (days 1-31 post-viral injection). To make accurate intake comparisons between dietary conditions, all food intake measurements were converted to kilocalories for analyses $(\mathrm{chow}=3.36 \mathrm{kcal} / \mathrm{g} ; \quad \mathrm{HFD}=5.24 \mathrm{kcal} / \mathrm{g})$. Two rats were 
excluded from behavioral analyses because of technical issues with daily measurements (1 CTR-KD/HFD, 1 CTRCtrl/Chow). After the conclusion of the behavioral study, rats were killed, and brains were rapidly removed and flashfrozen in $-70^{\circ} \mathrm{C}$ isopentane. VTA CTR-A knockdown was confirmed via qPCR, with rat GapDH (VIC-MGB) as the internal control.

\section{Statistical Analyses}

The $\alpha$ level was set at $p<0.05$ for all studies. Statistical analyses were performed using Statistica (StatSoft), GraphPad 5.0 (Prism), and Microsoft Excel. For FSCV studies, percent change in peak DA and post-session chow intake were compared using unpaired $t$-tests. For oral glucose tolerance testing and for all feeding studies, binned data were analyzed using separate mixed-design ANOVAs to account for the within-subjects experimental design, while assessing between-subjects effects (eg, drug treatment, AAV condition, dietary condition). Statistically significant effects were probed using Student-Newman-Keuls post hoc analyses, with the exception of the experiment testing the ability of IP sCT to promote negative energy balance in CTR-KD rats, in which Bonferroni post hoc comparisons were used to be conservative in the interpretation of the acute behavioral effects of the novel AAV. Finally, knockdown of CTR expression achieved with the novel AAV was analyzed by $t$-test for in vitro and preliminary in vivo testing, and by ANOVA with Student-Newman-Keuls post hoc analyses for the chronic dietary experiment.

\section{RESULTS}

\section{The CTR, the Core Signaling Component of the AmyR Complex, is Predominantly Expressed on VTA DA Neurons}

Given the potential involvement of DA signaling in mediating the energy balance effects of VTA AmyR signaling, we hypothesized that AmyR may be expressed on VTA DA neurons. The AmyR is a complex consisting of a dimer of the CTR and a receptor activity-modifying protein (Christopoulos et al, 1999; Young, 2005). Therefore, to analyze expression and colocalization of the AmyR on VTA DA neurons, we performed double-label IHC for CTR and the DA marker TH in the VTA. As shown in Figure 1, $62.6 \pm 6.1 \%$ of VTA CTR-expressing neurons co-express $\mathrm{TH}$; of the TH-positive neurons within the VTA, $11.8 \pm 3.6 \%$ co-express the CTR. This suggests that VTA AmyR activation may directly influence DA neurotransmission, pointing toward a potential mechanism by which VTA amylin could reduce food intake.

\section{Intra-VTA AmyR Activation Attenuates Food-Evoked Phasic DA Signaling in the NAc Core}

One of the major afferent targets of VTA DA neurons is the NAc (Fallon and Moore, 1978), and DA signaling within the core subregion (NACC) is important for food intake and reward (Brown et al, 2011; Vucetic and Reyes, 2010). To test whether VTA AmyR activation reduces NAcC DA, we performed FSCV to assess changes in food-evoked phasic
DA signaling in the NAcC associated with VTA AmyR activation. Indeed, food reward stimulates phasic increases in NAcC DA (Cone et al, 2014). Rats that were food restricted to $90 \%$ of their free-feeding body weight (McCutcheon et al, 2012) received random deliveries of a sucrose pellet, an event that reliably evoked a phasic increase in NAcC DA (see Figure 2a and b). The magnitude of DA evoked during retrieval of sucrose pellets was compared within-session, before and after unilateral intraVTA infusion of the AmyR agonist sCT $(0.04 \mu \mathrm{g}$; dose chosen from Mietlicki-Baase et al, 2013b) or vehicle (100 nl aCSF). Intra-VTA sCT significantly reduced peak NAcC DA evoked during pellet retrieval compared with vehicle infusion (Figure 2c; \% change from pre-infusion values, aCSF: $100.3 \pm 5.3 \%$, sCT $73.8 \pm 4.0 \% ; \mathrm{t}(7)=4.11, p<0.01$ ). Intra-VTA sCT also suppressed 30-min post-session home cage chow intake (Figure $2 \mathrm{~d} ; \mathrm{t}(7)=3.25, p<0.05$ ).

\section{NAcC DA Receptor Activation Attenuates the Chow Intake- and Body Weight-Suppressive Effects of VTA AmyR Activation}

The reduction in NAcC DA signaling observed in the FSCV studies likely corresponds with decreased DA receptor activation within the core. This led us to hypothesize that reduced NAcC DA receptor activation mediates VTA sCTinduced hypophagia. As both D1 and D2 receptors are present in the NAcC (Lu et al, 1998), we first established doses of the D1 agonist SKF-81297 (0.1, 0.6, $1.2 \mu \mathrm{g}$; vehicle, $200 \mathrm{nl} \mathrm{aCSF}$ ) and separately, the D2 agonist quinpirole (1.5, 3 , $6 \mu \mathrm{g}$; vehicle, $200 \mathrm{nl}$ aCSF) that are subthreshold for effects on chow intake when delivered unilaterally to the NAcC (SKF-81297, Supplementary Figures S1a and b, $\mathrm{F}_{3,15}=0.31, p=0.82$; quinpirole, Supplementary Figures S1c and d, $\mathrm{F}_{3,15}=0.19, p=0.90$ ) and 24 -h body weight gain (SKF-81297, $\mathrm{F}_{3,15}=1.00, p=0.42$; quinpirole, $\mathrm{F}_{3,15}=0.71$, $p=0.56)$. We also ensured that the combined NAcC administration of the D1 and D2 agonists (1.2 $\mu \mathrm{g}$ SKF81297 plus $6 \mu$ g quinpirole) was subthreshold for feeding or body weight effects (Supplementary Figure S1e and f; 24-h food: $F_{1,5}=0.002, p=0.96 ; 24$-h body weight: $F_{1,5}=0.16$, $p=0.71)$. This dose combination was then used to determine whether activating NAcC D1/D2 receptors could attenuate the reduction in food intake following VTA AmyR activation.

Separate chow-maintained rats received an intra-NAcC injection of the combined D1 and D2 receptor agonists $(1.2 \mu \mathrm{g}$ SKF-81297 and $6 \mu \mathrm{g}$ quinpirole; D1 + D2) or vehicle $(200 \mathrm{nl})$, followed by an intra-VTA injection of sCT $(0.04 \mu \mathrm{g})$ or vehicle $(100 \mathrm{nl})$. As shown in Figure 3a, activation of NAcC D1 and D2 receptors attenuated the reduction of chow intake produced by VTA AmyR activation (main effect of sCT $1,3,6$, and $24 \mathrm{~h}$, all ANOVAs $\mathrm{F}_{1,9} \geq 5.81$, $p \leq 0.04$; interaction between $\mathrm{D} 1+\mathrm{D} 2$ and $\mathrm{sCT}$ at $24 \mathrm{~h}$, $\mathrm{F}_{1,9}=7.99, p<0.02$; planned comparisons between vehicle/ sCT and D1 + D2/sCT at $24 \mathrm{~h}, p<0.05)$. NAcC DA receptor activation also inhibited the suppression of body weight produced by intra-VTA sCT (Figure 3b; interaction between $\mathrm{D} 1+\mathrm{D} 2$ and $\left.s \mathrm{CT} ; \mathrm{F}_{1,9}=13.01, p<0.01\right)$. These data indicate that decreased NACC D1/D2 receptor signaling mediates the intake- and body weight-suppressive effects of VTA AmyR activation. 

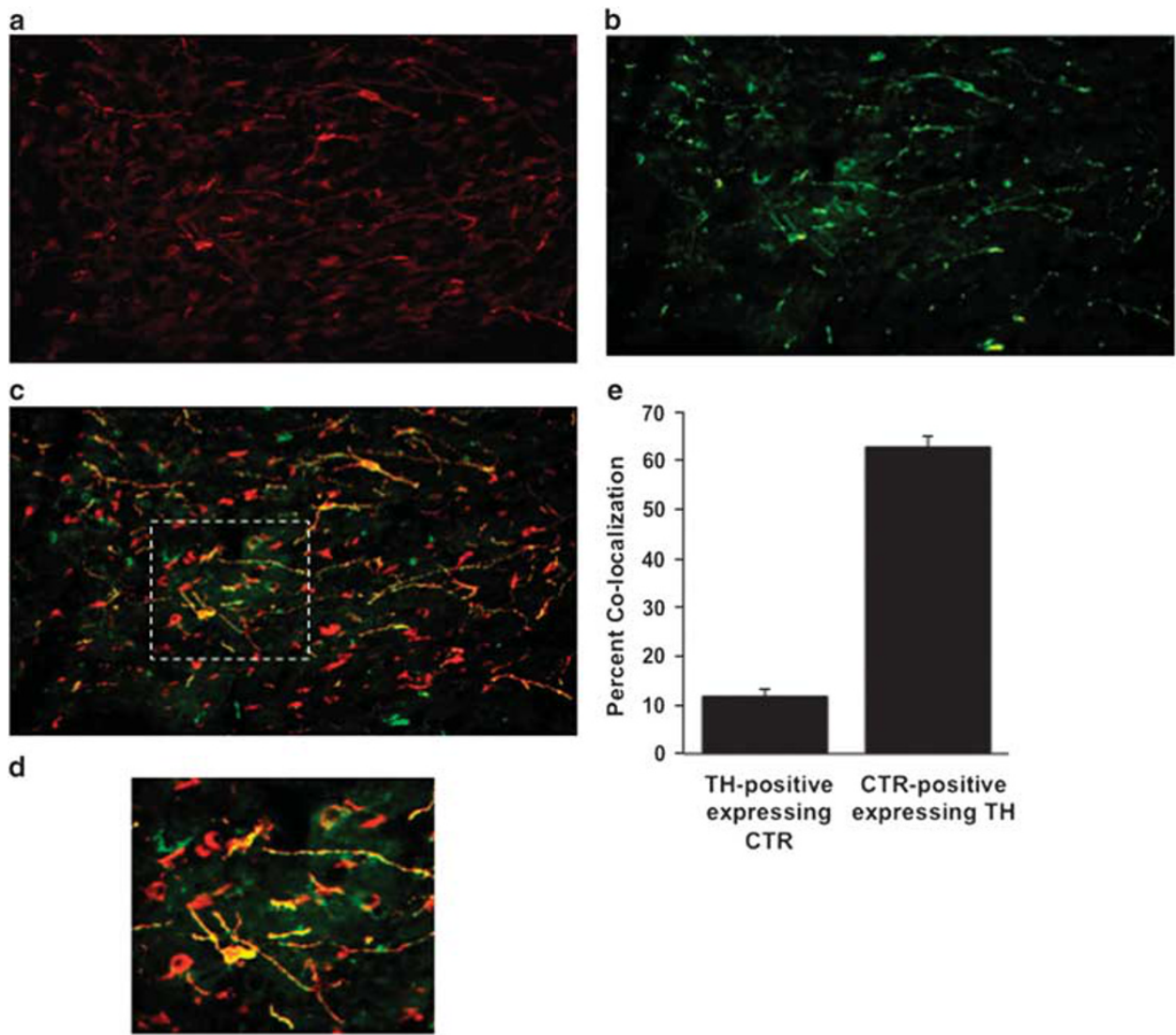

CTR

Figure I AmyRs are expressed on VTA DA neurons. Representative high-magnification $(\times 20)$ images from a coronal section containing the VTA show TH alone in red (a), CTR alone in green (b), and colocalized neurons expressing both TH and CTR (c). The dotted box in c is further magnified in d. Semiquantification of VTA neurons ( $n=6$ rats) demonstrated that approximately $63 \%$ of VTA CTR-expressing neurons also express TH, whereas approximately $12 \%$ of TH-positive neurons express the CTR (e). Data are expressed as mean \pm SEM.

\section{VTA AmyR Activation does not Produce CTA or Alter BG Levels}

Previous research from our laboratory showed that AmyR activation in the VTA did not induce pica (Mietlicki-Baase et al, 2013b), a measure of nausea/malaise in rodents (Andrews and Horn, 2006), suggesting that the intakesuppressive effects of VTA amylin are not caused by malaise. To rule out the possibility that VTA AmyR activation suppresses feeding by producing a conditioned avoidance response to food, rats underwent a two-bottle CTA experiment (Garcia et al, 1955; Kanoski et al, 2012). Two groups of rats received intra-VTA injection of aCSF $(\mathrm{CS}-; 100 \mathrm{nl})$ or sCT $(\mathrm{CS}+)$, with one group receiving sCT at a dose effective at reducing food intake in the VTA but subthreshold for effect when given ICV $(0.04 \mu \mathrm{g})$, and the other group receiving a higher dose of $\mathrm{sCT}$ that reduces food intake when delivered ICV $(0.4 \mu \mathrm{g})$. A third group of rats received IP injection of saline (CS-; $1 \mathrm{ml} / \mathrm{kg}$ ) or $\mathrm{LiCl}$ (CS +; $0.15 \mathrm{M})$, a substance known to elicit nausea/malaise in rodents (Kanoski et al, 2012; Spector et al, 1988), serving as a positive control for the CTA procedure. As shown in Figure $3 \mathrm{c}, \mathrm{LiCl}$-treated rats consumed significantly less of the $(\mathrm{CS}+)$-paired flavor than the $(\mathrm{CS}-)$-paired flavor during the test, indicating the presence of a CTA (interaction between drug treatment and flavor, $F_{2,22}=10.22$, $p<0.001$; within $\mathrm{LiCl}$ rats, $(\mathrm{CS}-)$-paired flavor $v s(\mathrm{CS}+)$ - paired flavor, $p<0.05)$. However, VTA AmyR activation did not produce a CTA, as consumption of (CS - )-paired and $(\mathrm{CS}+)$-paired flavors did not differ $(p>0.05$ for both $\mathrm{sCT}$ doses). These findings provide further evidence that the anorexia produced by VTA AmyR activation is independent of nausea/malaise and/or a conditioned avoidance to food.

As systemic administration of AmyR agonists lowers BG levels (Scherbaum, 1998) via action within the CNS (for a review, see Hayes et al, 2014; Lutz, 2005), it was important to determine whether VTA AmyR activation influences glycemia, an effect that could indirectly influence feeding. Accordingly, the effects of intra-VTA injections of sCT (0.004, 0.04, or $0.4 \mu \mathrm{g}$; vehicle, $100 \mathrm{nl})$ on BG were assessed in an oral glucose tolerance test. Intra-VTA sCT did not alter BG concentrations at any time (Figure 3d; all ANOVAs $\mathrm{F}_{3,18} \leq 2.27, p \geq 0.12$ ), suggesting that the intake-suppressive effects of VTA AmyR signaling are separable from glycemic changes, and that other AmyR-expressing CNS nuclei mediate the glycemic effects of amylin and AmyR agonists.

\section{VTA AmyR Activation Decreases Intake of a Palatable High-Fat Food by Reducing Meal Size}

Previous data show that intra-VTA sCT injection reduces intake of a palatable sucrose solution (Mietlicki-Baase et al, 2013b), but the effects of VTA AmyR activation on palatable 

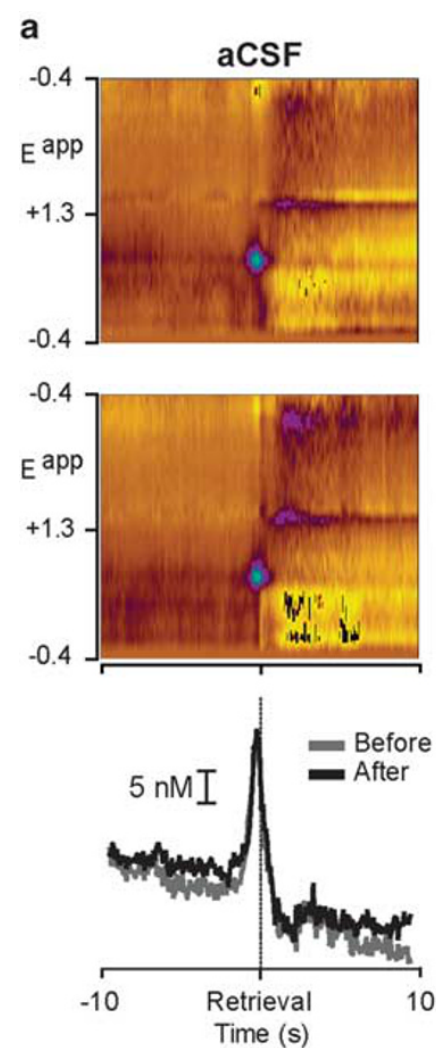

b
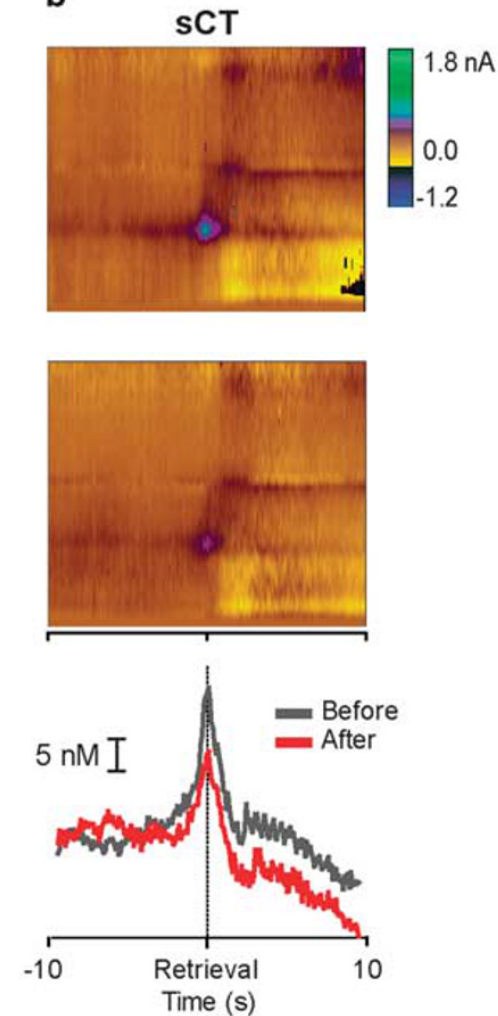

c

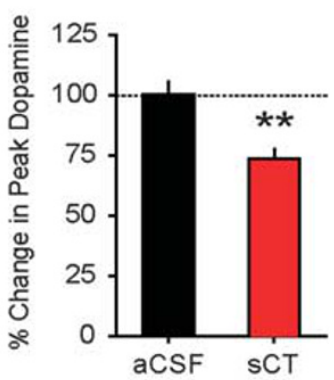

d

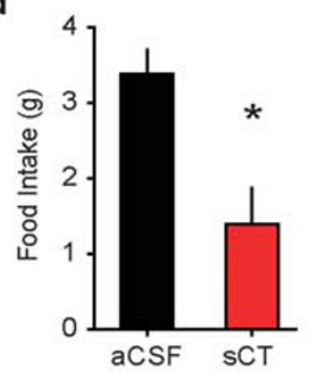

Figure 2 VTA AmyR activation decreases phasic DA in the NAcC evoked by food reward. (a) Top: average color plot ( 0 trials per rat) depicting current changes (color) as a function of electrode potential (y axis) in the $10 \mathrm{~s}$ before and after ( $\mathrm{x}$ axis) pellet retrieval. DA (identified by its oxidation ( $\sim+0.6 \mathrm{~V}$; green) and reduction ( - $0.2 \mathrm{~V}$; light yellow) features) was transiently evoked during pellet retrieval, before vehicle infusion. Middle: average color plot after VTA vehicle infusion. Bottom: average DA concentration aligned to retrieval before and after VTA aCSF extracted from individual color plots using chemometric analysis (Heien et al, 2004). (b) Average color plots before (top) and after VTA sCT (middle; 10 trials per rat). Average DA concentration aligned to pellet retrieval before and after VTA administration of the AmyR agonist sCT (bottom). (c) Percent change in NAcC peak DA evoked during pellet retrieval, following VTA vehicle or sCT. (d) Thirty-min post-session chow intake following intra-VTA vehicle or sCT. Bar data are mean \pm SEM; $n=9$; * $p<0.05 ;$ * ** $p<0.01$.

high-fat food intake are unknown. To test whether acute VTA AmyR activation reduces intake of a palatable HFD ( $60 \% \mathrm{kcal}$ from fat), rats were given intra-VTA injections of sCT $(0.01,0.04,0.4 \mu \mathrm{g})$ or vehicle $(100 \mathrm{nl})$ and HFD intake, meal patterns, and body weight were monitored for 24-h post-injection. Figure 4a shows a dose-dependent suppression of cumulative HFD intake by sCT (significant effects at $0.5,1,1.5,2,4,12$, and $24 \mathrm{~h}$; ANOVAs $\mathrm{F}_{3,27} \geq 3.00, p<0.05$ ). Meal pattern analyses reveal main effects of VTA sCT on meal size at all times (Figure $4 \mathrm{~b}$; all ANOVAs $\mathrm{F}_{3,27} \geq 3.02$, $p<0.05$ ), with fewer effects on meal number (Figure 4c; significant effects at $1,1.5,2,12$, and $24 \mathrm{~h}$; ANOVAs $\left.\mathrm{F}_{3,27} \geq 3.71, p \leq 0.02\right)$. The most robust reductions in meal size were observed with $0.4 \mu \mathrm{g} \mathrm{sCT}$, although all doses significantly reduced meal size compared with vehicle treatment at 12 and $24 \mathrm{~h}(p<0.05)$. Consistent with this finding, average meal duration over the 24 -h test period was also reduced by all doses of $s C T$ (in minutes, mean \pm SEM: vehicle, $15.88 \pm 1.87 ; 0.01 \mu \mathrm{g} \mathrm{sCT}, 8.79 \pm 1.70 ; 0.04 \mu \mathrm{g} \mathrm{sCT}$, $\left.11.59 \pm 1.31 ; 0.4 \mu \mathrm{g} \mathrm{sCT}, 8.05 \pm 1.62 ; \mathrm{F}_{3,27}=7.34, p<0.001\right)$. However, only the highest dose of sCT $(0.4 \mu \mathrm{g})$ reliably suppressed meal number $(0.4 \mu \mathrm{g} v s$ vehicle, $p<0.05$ at all listed times) or latency to consume the first meal (in minutes, mean \pm SEM: vehicle, $5.2 \pm 1.79 ; 0.01 \mu \mathrm{g}$ sCT, $58.53 \pm 45.26 ; \quad 0.04 \mu \mathrm{g} \quad \mathrm{sCT}, \quad 32.28 \pm 16.69 ; \quad 0.4 \mu \mathrm{g} \quad \mathrm{sCT}$,
$418.17 \pm 192.95 ; \mathrm{F}_{3,27}=4.03, p<0.02$; vehicle $v s 0.4 \mu \mathrm{g} \mathrm{sCT}$, $p<0.05)$. In addition, the highest dose of sCT reduced 24-h BW gain (Figure $4 \mathrm{~d} ; \mathrm{F}_{3,27}=8.11, p<0.001$ ). Together, these data indicate that the primary behavioral mechanism driving the reduction in HFD intake by VTA AmyR activation is suppression of meal size, rather than decreased meal number.

NAcC DA Receptor Agonism Attenuates the Intake- and Body Weight-Suppressive Effects of VTA AmyR Activation in HFD-Fed Rats

Current data show that the reductions in chow intake produced by intra-VTA sCT administration are attenuated by $\mathrm{NAcC}$ pretreatment with $\mathrm{DA}$ receptor agonists. To assess whether a similar DAergic mechanism mediates the suppression in HFD intake produced by VTA AmyR activation, we first confirmed that intra-NAcC administration of the highest doses of SKF-81297 $(1.2 \mu \mathrm{g})$ and quinpirole $(6 \mu \mathrm{g})$ tested in the chow studies did not alter intake or body weight gain in HFD-fed rats when administered either separately (Supplementary Figures S2a and b; food: $\mathrm{F}_{2,22}=0.89, p=0.42$; body weight: $\mathrm{F}_{2,22}=1.29$, $p=0.29$ ) or in combination (Supplementary Figure S2c and d; food: $F_{1,7}=0.15, p=0.71$; body weight: $F_{1,7}=0.06$, 
a

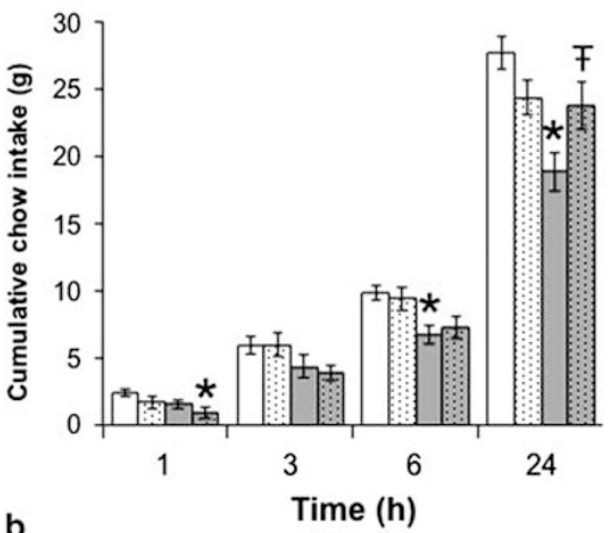

b

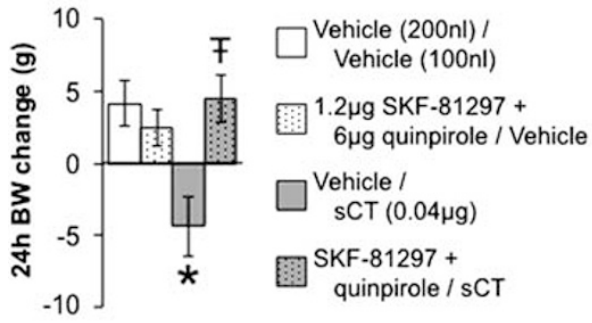

C
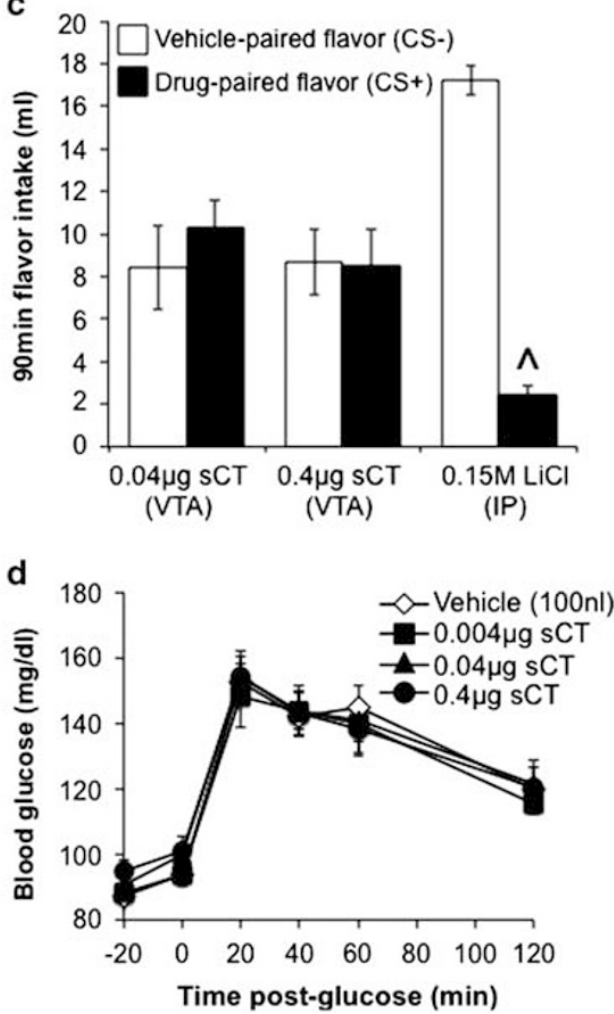

Figure 3 Activation of NAcC DA receptors with a combination of DI and D2 receptor agonists attenuates the energy balance effects of VTA AmyR activation in chow-fed rats. Direct intra-NAcC injection of a combination of SKF-8 297 (DI agonist) and quinpirole (D2 agonist) attenuates the reduction in chow intake (a) and 24-h body weight gain (b) produced by intra-VTA injection of sCT $(n=10)$. *Different from vehicle/vehicle $(p<0.05)$; ${ }^{\mathrm{F}}$ different from vehicle/sCT $(p<0.05)$. The key next to $b$ also applies to a. (c) Although IP injection of the known nausea/malaise-inducing agent LiCl produces CTA in rats, intra-VTA administration of SCT does not cause rats to exhibit CTA to the drug-paired flavor $\left(n=6-9\right.$ per stimulus condition). ${ }^{\wedge}$ Different from vehiclepaired flavor $(p<0.05)$. (d) VTA AmyR activation does not alter BG in an oral glucose tolerance test $(n=7)$. All data are shown as mean \pm SEM. See also Supplementary Figure SI.

$p=0.81)$. Next, we tested whether intra-NAcC injection of a combination of SKF-81297 plus quinpirole (D1 + D2; 1.2 and $6 \mu \mathrm{g}$, respectively; vehicle, $200 \mathrm{nl}$ ) would attenuate the hypophagia and body weight loss produced by intra-VTA sCT $(0.04 \mu \mathrm{g}$; vehicle, $100 \mathrm{nl})$ in HFD-fed rats. Figure $5 \mathrm{a}$ shows that VTA sCT potently suppressed HFD intake (main effect of sCT at 3,6 , and $24 \mathrm{~h}$; all ANOVAs $\mathrm{F}_{1,11} \geq 5.78$, $p \leq 0.04)$; however, intra-NAcC pretreatment with the $\mathrm{D} 1+\mathrm{D} 2$ agonists significantly attenuated these effects (interaction between D1 + D2/sCT at all times; all ANOVAs $\mathrm{F}_{1,11} \geq 4.96, p<0.05$; vehicle/sCT $v s \mathrm{D} 1+\mathrm{D} 2 / \mathrm{sCT}, p<0.05$ at 3,6 , and $24 \mathrm{~h}$ ). Similar results were observed for 24 -h body weight gain, as intra-NAcC D1 + D2 treatment attenuated the weight loss produced by intra-VTA sCT (Figure 5b; main effect of $\mathrm{sCT}$; $\mathrm{F}_{1,11}=6.33, p=0.03$; interaction between $\mathrm{D} 1+\mathrm{D} 2 / \mathrm{sCT}, \mathrm{F}_{1,11}=7.18, p=0.02$; vehicle/sCT $v s$ $\mathrm{D} 1+\mathrm{D} 2 / \mathrm{sCT}, p<0.05)$. These results indicate that the novel NAcC DA mechanism identified in the earlier chow study also mediates suppression of palatable food intake after VTA AmyR activation. NAcC D1/D2 activation attenuated the suppression of HFD intake by VTA sCT delivery at earlier times than observed in the chow study, suggesting the intriguing possibility that the changes in DA signaling induced by VTA AmyR signaling may have more impact on the intake of palatable compared with bland foods.

\section{A Novel AAV Decreases CTR Expression In Vitro and In Vivo}

To evaluate the effects of chronically reduced VTA AmyR signaling on energy balance, we developed an AAV to sitespecifically knockdown expression of CTR-A/B. We selected a potential sequence for shRNA interference-mediated CTR knockdown and tested its ability to reduce CTR expression in vitro in an immortalized rat neuronal cell line. Transient transfection of neuronal R-19 cells overexpressing CTR-A, the more highly expressed CTR subtype within the CNS (Young, 2005) and VTA (Mietlicki-Baase et al, 2013b), with the shRNA-CTR sequence produced a dramatic reduction $(>95 \%)$ in CTR-A mRNA expression (Figure 6a; $\mathrm{t}(2)=14.63, p<0.01)$, indicating the potential utility of this sequence for in vivo CTR knockdown. After packaging the sequence into an AAV (serotype I) co-expressing eGFP, we tested the efficacy of the AAV-shRNA in vivo by administering the AAV-CTR knockdown (CTR-KD) or a control AAV expressing eGFP (CTR-Ctrl) bilaterally into the VTA of rats. Consistent with the in vitro results, VTA CTR-KD produced robust $(>90 \%)$ knockdown of CTR-A mRNA in the VTA compared with CTR-Ctrl treatment (Figure 6b; $\mathrm{t}(7)=24.30, p<0.0001)$. VTA CTR-B expression was below threshold for significant detection in both CTR-Ctrl and 

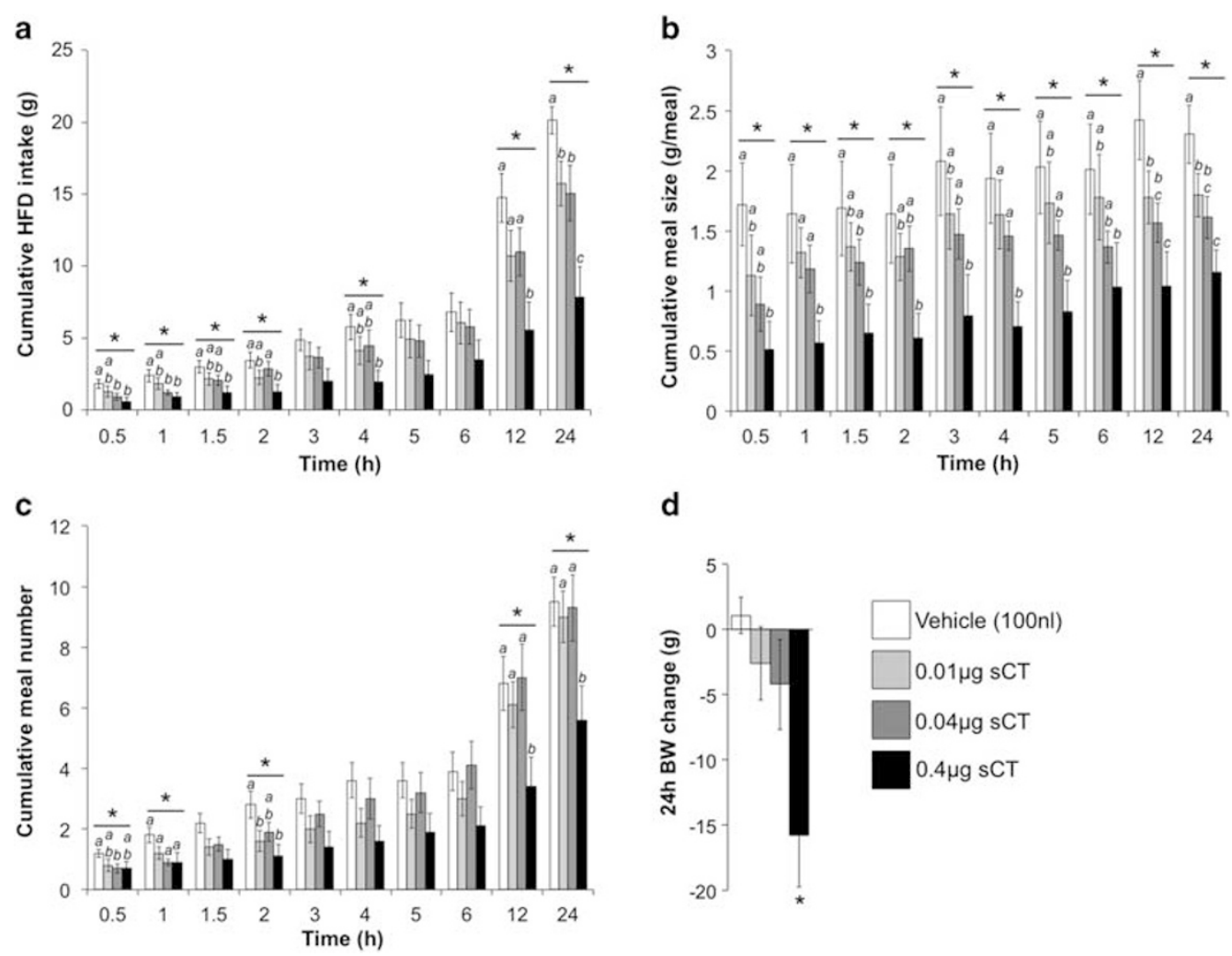

d

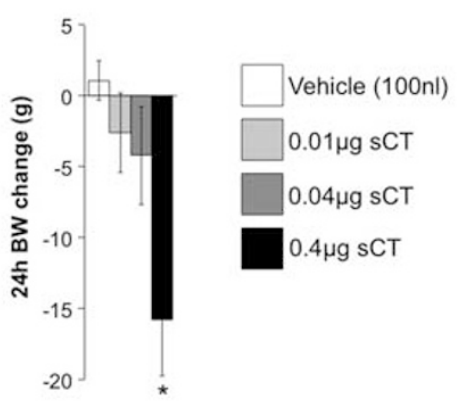

Figure 4 VTA AmyR activation suppresses intake of a palatable HFD primarily by decreasing meal size. Intra-VTA injection of sCT ( $n=10)$ reduced cumulative HFD consumption (a) and meal size (b), but only the highest dose of sCT decreased meal number (c). Body weight gain over the $24 \mathrm{~h}$ test was also reduced by $\mathrm{SCT}(\mathrm{d})$. *Main effect of $\mathrm{sCT}(p<0.05)$; within a time bin, bars with different letters are significantly different from each other $(p<0.05)$. The key next to $d$ applies to all panels. All data are shown as mean \pm SEM.

CTR-KD rats, consistent with previous data showing higher expression of CTR-A compared with CTR-B within the VTA (Mietlicki-Baase et al, 2013b).

\section{Knockdown of VTA AmyR by AAV-CTR-KD Blunts the Chow Intake- and Body Weight-Suppressive Effects of a Peripheral AmyR Agonist}

Peripheral sCT injection activates VTA AmyR to reduce chow intake and body weight (Mietlicki-Baase et al, 2013b). To confirm the behavioral relevance of VTA CTR-KD, chow-maintained CTR-KD and CTR-Ctrl rats received IP injection of sCT $(2 \mu \mathrm{g} / \mathrm{kg})$ or vehicle $(1 \mathrm{ml} / \mathrm{kg} 0.9 \% \mathrm{NaCl})$ and subsequent intake and body weight change were measured. In CTR-Ctrl rats, IP sCT potently suppressed food intake (Figure 6d; main effect of sCT at all times, all ANOVAs $\mathrm{F}_{1,14} \geq 9.58, p \leq 0.01$, planned comparisons between CTR-Ctrl/vehicle and CTR-Ctrl/sCT, $p<0.05$ at 3, 6, and $24 \mathrm{~h}$ ) and 24-h body weight gain (Figure 6e; main effect of sCT, $\mathrm{F}_{1,14}=8.09, p=0.01$; planned comparison between CTR-Ctrl/vehicle and CTR-Ctrl/sCT, $p<0.05$ ). In contrast, systemic sCT was ineffective at reducing food intake or body weight gain in VTA CTR-KD rats (all CTR-KD/vehicle vs CTR-KD/sCT planned comparisons, $p>0.05)$. AAV condition alone had no effect on feeding or body weight (CTR-Ctrl/vehicle vs CTR-KD/vehicle, all $p>0.05$ ) in this acute test. These data provide critical evidence supporting the VTA AmyR as a site-of-action for peripheral amylin agonists and show that reduction of CTR, and thus

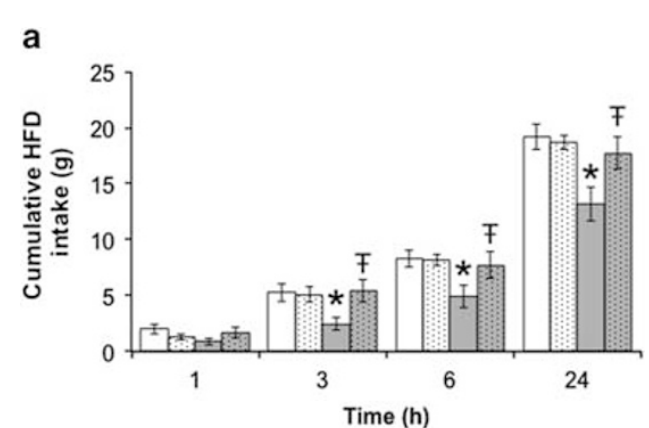

b

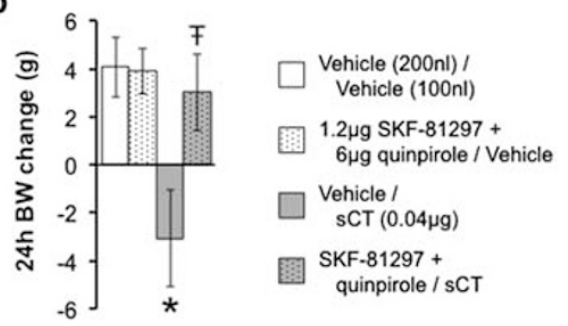

Figure 5 Intra-NAcC administration of a combination of DI and D2 receptor agonists blocks the energy balance effects of VTA AmyR activation in rats maintained on palatable HFD. Administration of SKF81297 (DI receptor agonist) plus quinpirole (D2 receptor agonist) directly to the NAcC blocks the suppression of HFD intake (a) and 24-h body weight gain (b) after intra-VTA sCT administration ( $n=12)$. *Different from vehicle/vehicle $(p<0.05)$; ${ }^{\mathrm{T}}$ different from vehicle/sCT $(p<0.05)$. The key next to $b$ applies to both panels. All data are shown as mean \pm SEM. See also Supplementary Figure S2. 
a

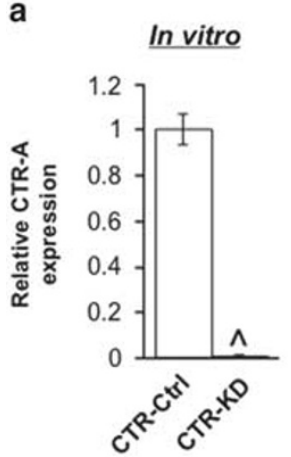

b

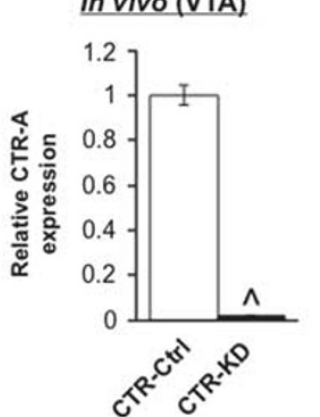

C
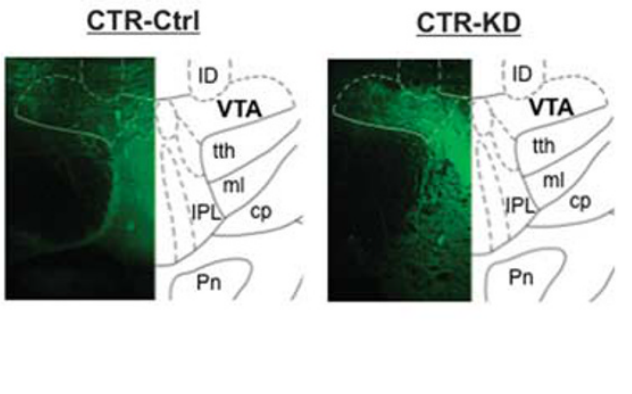

e

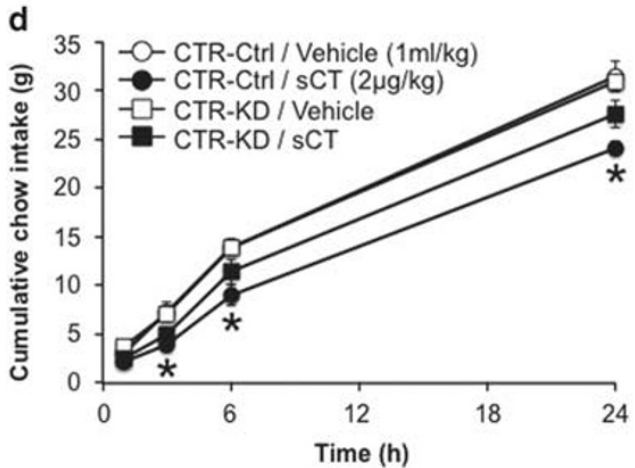


cells overexpressing CTR-A with a plasmid expressing the sequence targeting CTR mRNA decreased CTR expression ( $n=4$ ). (b) Injection of the CTR

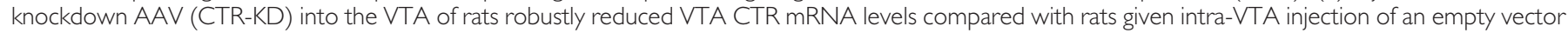

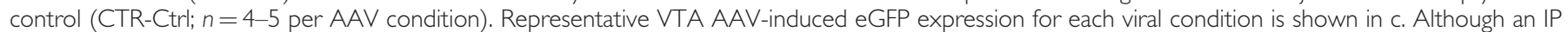

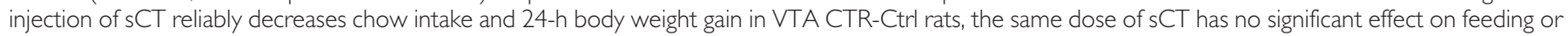

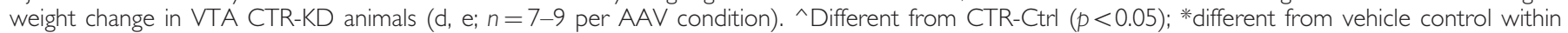
dietary condition $(p<0.05)$. All data are shown as mean \pm SEM.

functional AmyR, in the VTA has important acute behavioral consequences.

\section{Endogenous VTA AmyR Signaling is Required for the Long-Term Control of Energy Balance}

To assess the relevance of chronic endogenous VTA amylin signaling for energy balance control, rats were maintained on chow or on HFD; within each dietary condition, rats received either bilateral VTA CTR-KD or CTR-Ctrl AAV injections, resulting in a total of four experimental groups. Body weight was measured $24 \mathrm{~h}$ before viral injection to confirm that baseline values did not differ between groups (Supplementary Figure S3a; no effect of diet or planned AAV condition, no interaction; all $\left.\mathrm{F}_{1,22} \leq 3.38, p>0.05\right)$. Food intake was also monitored for $48 \mathrm{~h}$ before viral injection ( $-72 \mathrm{~h}$ to $-24 \mathrm{~h}$ ); although HFD-maintained rats consumed more kilocalories than chow-fed rats (Supplementary Figure S3b; main effect of diet, $\mathrm{F}_{1,22}=29.21, p<0.0001$ ), no differences were observed within dietary condition in rats that were to be assigned to each AAV condition (planned comparisons between CTR-Ctrl/Chow vs CTR-KD/Chow and CTR-Ctrl/HFD vs CTR-KD/HFD, $p>0.05)$.

Beginning the day after viral injection, food intake and body weight were recorded every $48 \mathrm{~h}$ to evaluate the longterm energy balance effects of chronically reduced VTA AmyR signaling in each dietary condition. In chow-fed rats, no differences in total body weight gain (Figure $7 \mathrm{c}$; main effects of diet and of AAV, all $F_{1,22} \geq 4.93, p \leq 0.04$; planned comparison between CTR-KD/Chow vs CTR-Ctrl/Chow, $p>0.05$ ) or total energy intake (Figure $7 \mathrm{~d}$; main effects of diet and of $\mathrm{AAV}$, all $\mathrm{F}_{1,22} \geq 8.31, p \leq 0.01$; planned comparison between CTR-KD/Chow vs CTR-Ctrl/Chow, $p>0.05)$ were observed. Regardless of viral condition, HFD-maintained rats gained more body weight and consumed more kilocalories over the course of the study than did chow-fed rats. However, HFD-fed CTR-KD rats showed an exacerbated hyperphagia and gained more total body weight than did HFD-fed CTR-Ctrl rats (planned comparisons of CTR-KD/HFD vs CTR-Ctrl/HFD for total energy intake and total body weight gain, all $p<0.05)$. The increased total kilocalorie intake and body weight gain results of HFD CTR-KD rats were recapitulated in 48-h measurements for the duration of the study (Figure $7 \mathrm{a}$ and b; kcal: main effect of AAV, main effect of diet, and/or diet $\times \mathrm{AAV}$ interaction for all bins except days $1-3$, all significant ANOVAs $\mathrm{F}_{1,22} \geq 4.44, p<0.05$; body weight: main effect of $\mathrm{AAV}$, main effect of diet, and/or diet $\times \mathrm{AAV}$ interaction for all days, all significant ANOVAs $F_{1,22} \geq 4.73$, $p<0.05)$. Effective AAV-mediated knockdown of VTA CTRA expression was confirmed by qPCR at the end of the study (Figure 7e; main effect of AAV; $\mathrm{F}_{1,24}=16.93, p<0.001$; planned comparisons between CTR-Ctrl/Chow vs CTR-KD/ Chow and CTR-Ctrl/HFD vs CTR-KD/HFD, $p<0.05$; no main effect of diet or diet $\times \mathrm{AAV}$ interaction, all $\mathrm{F}_{1,24} \leq 2.38$, $p>0.05)$. These data demonstrate a role for endogenous VTA amylin signaling in the long-term control of energy balance, particularly in rats fed a palatable HFD. 

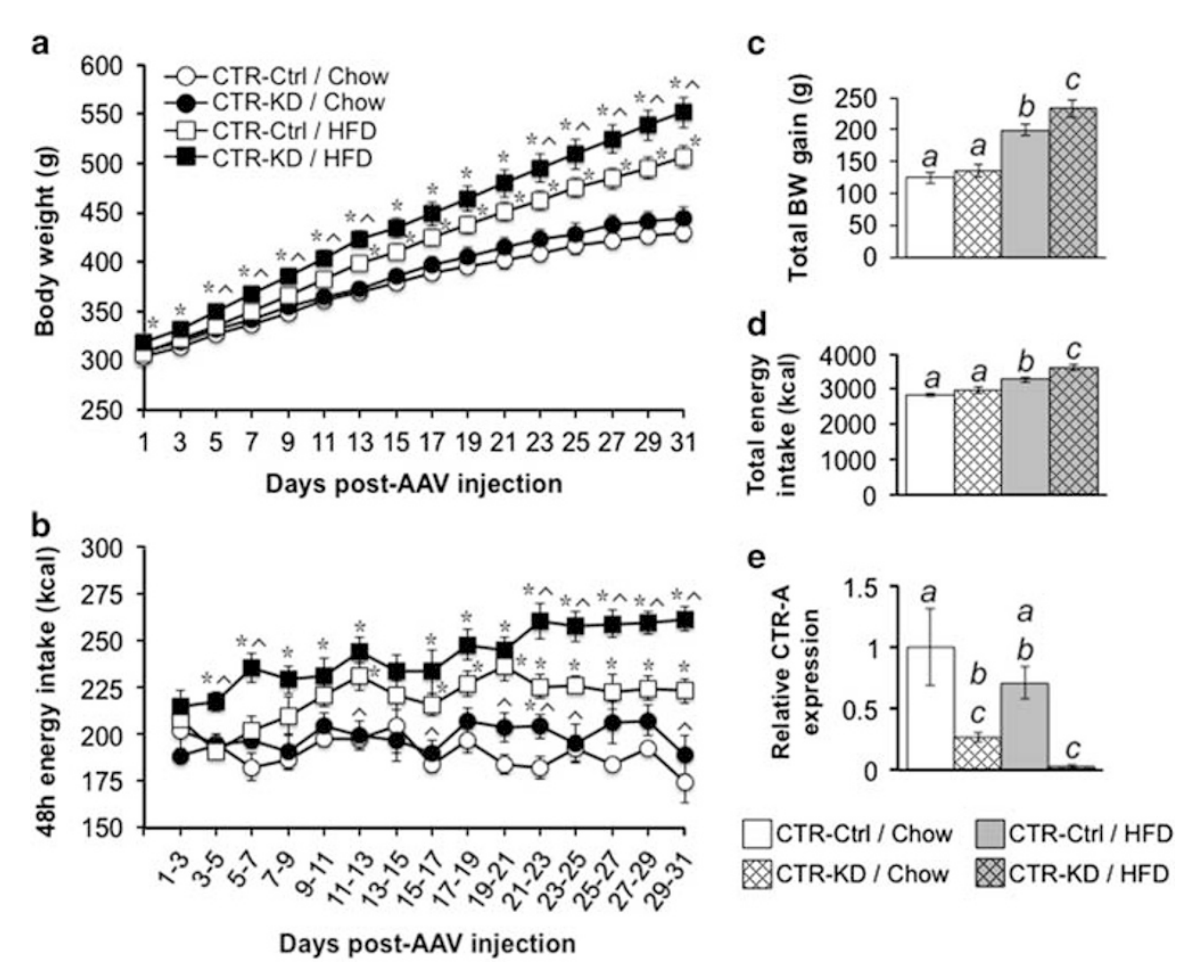

Figure 7 Endogenous amylin activates the VTA to provide long-term control of energy balance. Rats maintained on HFD or chow received intra-VTA injection of the AAV to knock down CTR expression (CTR-KD) or the control virus (CTR-Ctrl), and subsequent energy intake and body weight were monitored for I-month post-AAV injection ( $n=6-7$ per AAV/dietary condition). (a) HFD-fed VTA CTR-KD rats gained more body weight than any other treatment group; total body weight gain over the course of the experiment is shown in c. The enhanced body weight gain of HFD-fed CTR-KD rats is explained by hyperphagia, as rats in this condition consume more kilocalories than animals in any other treatment group both in 48-h measurements (b) and over the course of the study (d). VTA knockdown of CTR-A expression was confirmed via qPCR (e). For (a, b), *different from CTR-Ctrl/Chow ( $p<0.05$ ); ^different from CTR-Ctrl/HFD ( $p<0.05)$. The key in a also applies to b. For $(c-e)$, bars with different letters are significantly different from each other $(p<0.05)$; the key below e also applies to $c$ and d. All data are shown as mean \pm SEM. See also Supplementary Figure S3.

\section{DISCUSSION}

As amylin-based pharmacotherapies gain attention as potential treatments for obesity (Lutz, 2013; Roth et al, 2009; Sadry and Drucker, 2013), it is clear that a deeper comprehension of the distributed CNS effects of AmyR signaling is urgently needed. The existence of non-AP CNS nuclei that respond to amylin and amylin analogs, and the potential role of these sites in the regulation of feeding, has been particularly under-investigated. Only recently has the VTA been recognized as an important nucleus mediating the energy balance effects of amylin (Mietlicki-Baase et al, 2013b). The current data provide novel insight into a neuroanatomical mechanism mediating the energy balance effects of VTA AmyR activation, demonstrating that acute VTA AmyR activation produces hypophagia and weight loss by reducing DA signaling from the VTA to the NAcC. In addition, we provide the first evidence for site-specific, long-term control of energy balance by endogenous amylin, as AAV-mediated knockdown of VTA AmyR resulted in hyperphagia and enhanced body weight gain in HFD-fed rats.

The fact that VTA AmyR activation reduces palatable food intake and motivation to obtain a palatable food (Mietlicki-Baase et al, 2013b), together with the established role of VTA DA signaling in the regulation of feeding and reward, highlighted the possible involvement of DA in the energy balance effects of VTA amylin. We focused our attention on DA signaling from the VTA to the NAcC, a pathway known to influence food intake and motivated behavior (Cone et al, 2014; Roitman et al, 2004; Vucetic and Reyes, 2010; Wanat et al, 2010). Several feeding-related peptides modulate VTA to NAc DA neurotransmission, including leptin (Krugel et al, 2003) and ghrelin (Cone et al, 2014; Skibicka et al, 2013). Here, we found that VTA AmyR activation reduces NAcC D1/D2 receptor activation to decrease feeding. Our FSCV data suggest that this is likely due to VTA amylin-induced suppression of DA release in the NAcC. Interestingly, the ability of NAcC D1/D2 receptor activation to attenuate the energy balance effects of VTA sCT was observed both in rats that were fed chow or a palatable HFD, highlighting a common DA-mediated mechanism by which VTA AmyR activation reduces intake of both bland and palatable foods.

VTA AmyR activation with sCT produces acute reductions in ad libitum intake of a palatable $15 \%$ sucrose solution (Mietlicki-Baase et al, 2013b). Here, we show that intra-VTA $\mathrm{SCT}$ also suppresses HFD intake, indicating a broader role for VTA amylin signaling in the control of palatable food intake. Interestingly, VTA AmyR-mediated suppression of sucrose intake and of HFD intake occurs at a dose of sCT $(0.01 \mu \mathrm{g})$ that is ineffective at significantly reducing intake of chow (Mietlicki-Baase et al, 2013b). Although VTA AmyR activation may more potently suppress intake of palatable foods compared with less palatable chow, intra-VTA sCT-induced reductions in chow and in HFD intake are both mediated primarily by reductions in meal size rather than meal number, consistent 
with systemic amylin's satiating properties (Bello et al, 2008; Lutz, 2005; Lutz et al, 1995b). Along with the current findings indicating a role for NAcC DA signaling in VTA amylin-mediated control of feeding, these data suggest that VTA AmyR activation may engage common physiological (DA) and behavioral (meal size) mechanisms to produce the suppression of intake of both palatable and bland foods by VTA AmyR activation. These data also raise the point that VTA amylin signaling may exert more robust anorectic effects on palatable ingesta. A similar possibility is that amylin may have stronger anorectic effects with regard to non-homeostatic/hedonic feeding. A previous report from our laboratory showed that VTA AmyR activation can reduce the motivation to obtain a palatable food, as measured by operant responding for sucrose pellets in a progressive ratio task (Mietlicki-Baase et al, 2013b). Although operant responding for HFD has not been evaluated, it is possible that issues of food reward/ motivation and not simply palatability may also impact the ability of VTA amylin to suppress HFD intake.

VTA DA neurons project to several other feeding-relevant CNS sites besides the NAcC, including the NAc shell (Fallon and Moore, 1978), the prefrontal cortex (Berger et al, 1976), the central nucleus of the amygdala (Fallon et al, 1978; Mogenson and $\mathrm{Wu}, 1982$ ), and the hippocampus (Scatton et al, 1980). The ability of VTA AmyR signaling to modulate DA signaling to these other sites remains untested, but represents an extremely important extension of the current results. For example, DA signaling in the NAc shell is relevant for many aspects of feeding and food reward (Baldo and Kelley, 2007; Bassareo and Di Chiara, 1999; Beyene et al, 2010). The dopaminergic projection from the VTA to the shell may also contribute to the intake-suppressive effects of VTA AmyR activation. Indeed, elucidating the impact of VTA AmyR activation on DA release in the shell would be an intriguing first step toward addressing a possible role of the VTA-NAc shell connection in the ability of VTA amylin to promote negative energy balance. In addition, as amylin binding is observed in many CNS nuclei (Christopoulos et al, 1995; Paxinos et al, 2004; Sexton et al, 1994), it is likely that sites other than the VTA contribute directly or indirectly to the effects of amylin not only for feeding but for other physiological effects such as glycemic control. Our current data support this notion as VTA AmyR activation with sCT did not change BG levels in an oral glucose tolerance test, suggesting that other CNS site(s) mediate the ability of amylin to regulate glycemia (Hayes et al, 2014).

In addition, although VTA CTR are highly expressed on DA neurons, it is clear that a percentage of VTA CTRexpressing cells do not colocalize with TH-positive neurons. The VTA contains sizable non-DA neuronal populations (eg, glutamate, GABA) (Dobi et al, 2010), and testing the potential co-expression of the AmyR with different neurotransmitter phenotypes may provide clues as to other mechanisms involved in the acute energy balance effects of VTA amylin signaling. Although the energy balance effects of VTA sCT are almost completely reversed by NAc core administration of DA receptor agonists in HFD-maintained rats, sCT-induced hypophagia and body weight loss are attenuated but not reversed by intra-core $\mathrm{D} 1+\mathrm{D} 2$ receptor agonism in chow-fed rats. This suggests that the ability of VTA AmyR activation to promote negative energy balance in chow-fed animals may also involve DA-independent mechanisms or processing of DA signaling in other CNS nuclei. In contrast, in HFD-maintained animals the hypophagic effects of VTA AmyR signaling appear to rely more heavily on DA signaling in the NAc core. Thus, it is certainly possible that VTA AmyR activation may engage additional neurotransmitter systems other than DA to partially mediate the anorectic effects of VTA AmyR activation. At present, with the absence of widely accepted and validated antibodies for these other neurotransmitter systems, future studies using transgenic fluorescent-reporter mice and neuropharmacological analyses will be needed to further such explorations.

There is a paucity of data in the literature examining the role of the amylin system in the chronic regulation of feeding and body weight. The limited number of energy balance studies in amylin knockout mice demonstrate only minimal phenotypic alterations, with no effect on food intake and minimal or no increase in body weight (Dacquin et al, 2004; Gebre-Medhin et al, 1998; Lutz, 2005; Olsson et al, 2012). In lean chow-fed rats, chronic systemic administration of the AmyR antagonist AC187 has no effect on feeding or body weight (Grabler and Lutz, 2004), although in contrast, chronic central AC187 administration increased food intake and adiposity (Rushing et al, 2001). Together, these previous reports indicate that a centrally targeted manipulation of the amylin system induces more potent energy balance effects. Here, we chose a novel and deductive approach to examine the long-term role of endogenous central amylin signaling in control of energy balance by site-specifically and chronically knocking down AmyR signaling in the VTA. Data show that VTA AmyR can be directly activated by systemic amylin administration, and furthermore, that endogenous amylin signaling in the VTA is involved in the control of energy balance. Interestingly, VTA CTR-KD-mediated increases in feeding and weight gain were observed only in rats maintained on HFD; chowfed CTR-KD animals were nearly identical to chow-fed AAV controls in their energy intake and body weight. The earlier finding that chow intake was increased by chronic central AmyR blockade (Rushing et al, 2001) contrasts with these VTA CTR-KD data. However, the previous study used icv administration of the AmyR antagonist (Rushing et al, 2001) as opposed to the site-specific knockdown of AmyR signaling in the current experiment. This emphasizes the intriguing possibility that other CNS sites, likely including the AP, contribute more strongly to the chow intakesuppressive effects of amylin, whereas the VTA has more robust effects on more palatable food. Importantly, the newly developed CTR-AAV will allow for the investigation of long-term energy balance control by amylin signaling in other isolated CNS sites, such as the AP, providing the opportunity to gain a clearer understanding of the site- and diet-specific effects of AmyR signaling.

The discovery that VTA amylin-induced hypophagia and body weight loss is mediated by decreased NAcC DA signaling is a critical first step in understanding the downstream neuroanatomical targets and mechanisms engaged by VTA AmyR activation. Furthermore, the longterm control of palatable food intake by VTA amylin, identified in our CTR-KD studies, represents a new technology for CNS amylin research. Collectively, these 
results provide evidence for novel behavioral and neuroanatomical mechanisms mediating the regulation of energy balance by VTA AmyR signaling. As VTA amylin signaling exerts both acute and chronic control over body weight and palatable food intake, the current findings add support to the notion that amylin-based compounds may be effective pharmacotherapies for the treatment of obesity.

\section{FUNDING AND DISCLOSURE}

The authors declare no conflict of interest.

\section{ACKNOWLEDGEMENTS}

We thank Thomas Lutz for guidance in CTR IHC, Heath Schmidt for technical advice on D1/D2 agonist studies, and the University of Pennsylvania Viral Core for their expertise in developing the CTR shRNA-AAV. Valuable technical assistance was provided by Marco Guevara, Kieran KochLaskowski, Daniela Mendez, Orianne Montaubin, Chan Nguyen, Amit Pujari, and Christopher Turner. This research was supported by NIH-DK097954 (EGM-B), DA025634 (MFR), and DK096139 (MRH).

\section{REFERENCES}

Andrews PL, Horn CC (2006). Signals for nausea and emesis: implications for models of upper gastrointestinal diseases. Auton Neurosci 125: 100-115.

Baik JH (2013). Dopamine signaling in reward-related behaviors. Front Neural Circuits 7: 152.

Baldo BA, Kelley AE (2007). Discrete neurochemical coding of distinguishable motivational processes: insights from nucleus accumbens control of feeding. Psychopharmacology (Berl) 191: 439-459.

Banks WA, Kastin AJ (1998). Differential permeability of the blood-brain barrier to two pancreatic peptides: insulin and amylin. Peptides 19: 883-889.

Banks WA, Kastin AJ, Maness LM, Huang W, Jaspan JB (1995). Permeability of the blood-brain barrier to amylin. Life Sci 57: 1993-2001.

Bassareo V, Di Chiara G (1999). Differential responsiveness of dopamine transmission to food-stimuli in nucleus accumbens shell/core compartments. Neuroscience 89: 637-641.

Bello NT, Kemm MH, Moran TH (2008). Salmon calcitonin reduces food intake through changes in meal sizes in male rhesus monkeys. Am J Physiol Regul Integr Comp Physiol 295: R76-R81.

Bence KK, Delibegovic M, Xue B, Gorgun CZ, Hotamisligil GS, Neel BG et al (2006). Neuronal PTP1B regulates body weight, adiposity and leptin action. Nat Med 12: 917-924.

Berger B, Thierry AM, Tassin JP, Moyne MA (1976). Dopaminergic innervation of the rat prefrontal cortex: a fluorescence histochemical study. Brain Res 106: 133-145.

Beyene M, Carelli RM, Wightman RM (2010). Cue-evoked dopamine release in the nucleus accumbens shell tracks reinforcer magnitude during intracranial self-stimulation. Neuroscience 169: 1682-1688.

Brown HD, McCutcheon JE, Cone JJ, Ragozzino ME, Roitman MF (2011). Primary food reward and reward-predictive stimuli evoke different patterns of phasic dopamine signaling throughout the striatum. Eur J Neurosci 34: 1997-2006.

Chapman I, Parker B, Doran S, Feinle-Bisset C, Wishart J, Strobel S et al (2005). Effect of pramlintide on satiety and food intake in obese subjects and subjects with type 2 diabetes. Diabetologia 48: 838-848.
Christopoulos G, Paxinos G, Huang XF, Beaumont K, Toga AW, Sexton PM (1995). Comparative distribution of receptors for amylin and the related peptides calcitonin gene related peptide and calcitonin in rat and monkey brain. Can J Physiol Pharmacol 73: 1037-1041.

Christopoulos G, Perry KJ, Morfis M, Tilakaratne N, Gao Y, Fraser NJ et al (1999). Multiple amylin receptors arise from receptor activity-modifying protein interaction with the calcitonin receptor gene product. Mol Pharmacol 56: 235-242.

Cone JJ, Chartoff EH, Potter DN, Ebner SR, Roitman MF (2013). Prolonged high fat diet reduces dopamine reuptake without altering DAT gene expression. PLoS One 8: e58251.

Cone JJ, McCutcheon JE, Roitman MF (2014). Ghrelin acts as an interface between physiological state and phasic dopamine signaling. J Neurosci 34: 4905-4913.

Dacquin R, Davey RA, Laplace C, Levasseur R, Morris HA, Goldring SR et al (2004). Amylin inhibits bone resorption while the calcitonin receptor controls bone formation in vivo. $J$ Cell Biol 164: 509-514.

Dobi A, Margolis EB, Wang HL, Harvey BK, Morales M (2010). Glutamatergic and nonglutamatergic neurons of the ventral tegmental area establish local synaptic contacts with dopaminergic and nondopaminergic neurons. J Neurosci 30: 218-229.

Domingos AI, Vaynshteyn J, Voss HU, Ren X, Gradinaru V, Zang F et al (2011). Leptin regulates the reward value of nutrient. Nat Neurosci 14: 1562-1568.

Fallon JH, Koziell DA, Moore RY (1978). Catecholamine innervation of the basal forebrain. II. Amygdala, suprarhinal cortex and entorhinal cortex. J Comp Neurol 180: 509-532.

Fallon JH, Moore RY (1978). Catecholamine innervation of the basal forebrain. IV. Topography of the dopamine projection to the basal forebrain and neostriatum. J Comp Neurol 180: 545-580.

Garcia J, Kimeldorf DJ, Koelling RA (1955). Conditioned aversion to saccharin resulting from exposure to gamma radiation. Science 122: 157-158.

Gebre-Medhin S, Mulder H, Pekny M, Westermark G, Tornell J, Westermark P et al (1998). Increased insulin secretion and glucose tolerance in mice lacking islet amyloid polypeptide (amylin). Biochem Biophys Res Commun 250: 271-277.

Grabler V, Lutz TA (2004). Chronic infusion of the amylin antagonist AC 187 increases feeding in Zucker fa/fa rats but not in lean controls. Physiol Behav 81: 481-488.

Grill HJ (2006). Distributed neural control of energy balance: contributions from hindbrain and hypothalamus. Obesity 14(Suppl 5): 216S-221S.

Grill HJ, Kaplan JM (2002). The neuroanatomical axis for control of energy balance. Front Neuroendocrinol 23: 2-40.

Hayes MR, Mietlicki-Baase EG, Kanoski SE, De Jonghe BC (2014). Incretins and amylin: neuroendocrine communication between the gut, pancreas, and brain in control of food intake and blood glucose. Annu Rev Nutr 34: 3.1-3.24.

Heien ML, Johnson MA, Wightman RM (2004). Resolving neurotransmitters detected by fast-scan cyclic voltammetry. Anal Chem 76: 5697-5704.

Ikemoto S (2010). Brain reward circuitry beyond the mesolimbic dopamine system: a neurobiological theory. Neurosci Biobehav Rev 35: 129-150.

Johnson KH, O’Brien TD, Hayden DW, Jordan K, Ghobrial HK, Mahoney WC et al (1988). Immunolocalization of islet amyloid polypeptide (IAPP) in pancreatic beta cells by means of peroxidase-antiperoxidase (PAP) and protein A-gold techniques. Am J Pathol 130: 1-8.

Kahn SE, D'Alessio DA, Schwartz MW, Fujimoto WY, Ensinck JW, Taborsky GJ Jr et al (1990). Evidence of cosecretion of islet amyloid polypeptide and insulin by beta-cells. Diabetes 39: 634-638.

Kanoski SE, Rupprecht LE, Fortin SM, De Jonghe BC, Hayes MR (2012). The role of nausea in food intake and body weight 
suppression by peripheral GLP-1 receptor agonists, exendin-4 and liraglutide. Neuropharmacology 62: 1916-1927.

Krugel U, Schraft T, Kittner H, Kiess W, Illes P (2003). Basal and feeding-evoked dopamine release in the rat nucleus accumbens is depressed by leptin. Eur J Pharmacol 482: 185-187.

Lu XY, Ghasemzadeh MB, Kalivas PW (1998). Expression of D1 receptor, D2 receptor, substance $\mathrm{P}$ and enkephalin messenger RNAs in the neurons projecting from the nucleus accumbens. Neuroscience 82: 767-780.

Lucas F, Sclafani A (1996). Capsaicin attenuates feeding suppression but not reinforcement by intestinal nutrients. Am J Physiol 270: R1059-R1064.

Lutz TA (2005). Pancreatic amylin as a centrally acting satiating hormone. Curr Drug Targets 6: 181-189.

Lutz TA (2013). The interaction of amylin with other hormones in the control of eating. Diabetes Obes Metab 15: 99-111.

Lutz TA, Del Prete E, Scharrer E (1994). Reduction of food intake in rats by intraperitoneal injection of low doses of amylin. Physiol Behav 55: 891-895.

Lutz TA, Del Prete E, Scharrer E (1995a). Subdiaphragmatic vagotomy does not influence the anorectic effect of amylin. Peptides 16: 457-462.

Lutz TA, Geary N, Szabady MM, Del Prete E, Scharrer E (1995b). Amylin decreases meal size in rats. Physiol Behav 58: 1197-1202.

Lutz TA, Senn M, Althaus J, Del Prete E, Ehrensperger F, Scharrer E (1998). Lesion of the area postrema/nucleus of the solitary tract (AP/NTS) attenuates the anorectic effects of amylin and calcitonin gene-related peptide (CGRP) in rats. Peptides 19: 309-317.

Lutz TA, Tschudy S, Rushing PA, Scharrer E (2000). Amylin receptors mediate the anorectic action of salmon calcitonin (sCT). Peptides 21: 233-238.

McCutcheon JE, Beeler JA, Roitman MF (2012). Sucrose-predictive cues evoke greater phasic dopamine release than saccharinpredictive cues. Synapse 66: 346-351.

Mietlicki-Baase EG, Hayes MR (2014). Amylin activates distributed CNS nuclei to control energy balance. Physiol Behav; doi:10.1016.j.physbeh.2014.01.013 (epub ahead of print 28 January 2014).

Mietlicki-Baase EG, Ortinski PI, Rupprecht LE, Olivos DR, Alhadeff AL, Pierce RC et al (2013a). The food intake-suppressive effects of glucagon-like peptide- 1 receptor signaling in the ventral tegmental area are mediated by AMPA/kainate receptors. Am J Physiol Endocrinol Metab 305: E1367-E1374.

Mietlicki-Baase EG, Rupprecht LE, Olivos DR, Zimmer DJ, Alter $\mathrm{MD}$, Pierce RC et al (2013b). Amylin receptor signaling in the ventral tegmental area is physiologically relevant for the control of food intake. Neuropsychopharmacology 38: 1685-1697.

Mogenson GJ, Wu M (1982). Electrophysiological and behavioral evidence of interaction of dopaminergic and gustatory afferents in the amygdala. Brain Res Bull 8: 685-691.

Mollet A, Gilg S, Riediger T, Lutz TA (2004). Infusion of the amylin antagonist AC 187 into the area postrema increases food intake in rats. Physiol Behav 81: 149-155.

Narayanan NS, Guarnieri DJ, DiLeone RJ (2010). Metabolic hormones, dopamine circuits, and feeding. Front Neuroendocrinol 31: 104-112.

Olsson M, Herrington MK, Reidelberger RD, Permert J, GebreMedhin S, Arnelo U (2012). Food intake and meal pattern in IAPP knockout mice with and without infusion of exogenous IAPP. Scand J Gastroenterol 47: 191-196.

Paxinos G, Chai SY, Christopoulos G, Huang XF, Toga AW, Wang HQ et al (2004). In vitro autoradiographic localization of calcitonin and amylin binding sites in monkey brain. J Chem Neuroanat 27: 217-236.

Paxinos G, Watson C (2006). The Rat Brain in Stereotaxic Coordinates. 6th edn, Elsevier Academic Press: Burlington, MA.

Phillips PE, Johns JM, Lubin DA, Budygin EA, Gainetdinov RR, Lieberman JA et al (2003). Presynaptic dopaminergic function is largely unaltered in mesolimbic and mesostriatal terminals of adult rats that were prenatally exposed to cocaine. Brain Res 961: 63-72.

Roitman MF, Stuber GD, Phillips PE, Wightman RM, Carelli RM (2004). Dopamine operates as a subsecond modulator of food seeking. J Neurosci 24: 1265-1271.

Roth JD, Maier H, Chen S, Roland BL (2009). Implications of amylin receptor agonism: integrated neurohormonal mechanisms and therapeutic applications. Arch Neurol 66: 306-310.

Rushing PA, Hagan MM, Seeley RJ, Lutz TA, D’Alessio DA, Air EL et al (2001). Inhibition of central amylin signaling increases food intake and body adiposity in rats. Endocrinology 142: 5035.

Rushing PA, Hagan MM, Seeley RJ, Lutz TA, Woods SC (2000). Amylin: a novel action in the brain to reduce body weight. Endocrinology 141: 850-853.

Sadry SA, Drucker DJ (2013). Emerging combinatorial hormone therapies for the treatment of obesity and T2DM. Nat Rev Endocrinol 9: 425-433.

Scatton B, Simon H, Le Moal M, Bischoff S (1980). Origin of dopaminergic innervation of the rat hippocampal formation. Neurosci Lett 18: 125-131.

Scherbaum WA (1998). The role of amylin in the physiology of glycemic control. Exp Clin Endocrinol Diabetes 106: 97-102.

Schmidt HD, Pierce RC (2006). Cooperative activation of D1-like and D2-like dopamine receptors in the nucleus accumbens shell is required for the reinstatement of cocaine-seeking behavior in the rat. Neuroscience 142: 451-461.

Sexton PM, Paxinos G, Kenney MA, Wookey PJ, Beaumont K (1994). In vitro autoradiographic localization of amylin binding sites in rat brain. Neuroscience 62: 553-567.

Sinkala E, McCutcheon JE, Schuck MJ, Schmidt E, Roitman MF, Eddington DT (2012). Electrode calibration with a microfluidic flow cell for fast-scan cyclic voltammetry. Lab Chip 12: 2403-2408. Skibicka KP, Shirazi RH, Rabasa-Papio C, Alvarez-Crespo M, Neuber C, Vogel H et al (2013). Divergent circuitry underlying food reward and intake effects of ghrelin: dopaminergic VTAaccumbens projection mediates ghrelin's effect on food reward but not food intake. Neuropharmacology 73: 274-283.

Smith SR, Blundell JE, Burns C, Ellero C, Schroeder BE, Kesty NC et al (2007). Pramlintide treatment reduces 24 -h caloric intake and meal sizes and improves control of eating in obese subjects: a 6-wk translational research study. Am J Physiol Endocrinol Metab 293: E620-E627.

Spector AC, Breslin P, Grill HJ (1988). Taste reactivity as a dependent measure of the rapid formation of conditioned taste aversion: a tool for the neural analysis of taste-visceral associations. Behav Neurosci 102: 942-952.

Swanson CJ, Heath S, Stratford TR, Kelley AE (1997). Differential behavioral responses to dopaminergic stimulation of nucleus accumbens subregions in the rat. Pharmacol Biochem Behav 58: 933-945.

Vucetic Z, Reyes TM (2010). Central dopaminergic circuitry controlling food intake and reward: implications for the regulation of obesity. Wiley Interdiscip Rev Syst Biol Med 2: 577-593.

Wanat MJ, Kuhnen CM, Phillips PE (2010). Delays conferred by escalating costs modulate dopamine release to rewards but not their predictors. J Neurosci 30: 12020-12027.

Young A (2005). Receptor pharmacology. Adv Pharmacol 52: 47-65.

Supplementary Information accompanies the paper on the Neuropsychopharmacology website (http://www.nature.com/npp) 